\title{
1 Structural basis for acyl chain control over glycosphingolipid sorting and vesicular trafficking
}

3 Stefanie S. Schmieder ${ }^{1}$, Raju Tatituri ${ }^{2,3}$, Michael Anderson ${ }^{1,4}$, Kate Kelly ${ }^{1}$, and Wayne I.

4 Lencer $^{1,3,4^{*}}$

51 Division of Gastroenterology, Boston Children's Hospital, Boston MA 02115

62 Division of Rheumatology, Brigham and Women's Hospital, Boston MA 02115

73 Harvard Medical School, Boston MA 02115

84 Harvard Digestive Diseases Center, Boston, MA 02115

$9 \quad *$ Corresponding author

\section{Abstract}

12 The complex sphingolipids exhibit a diversity of ceramide acyl chain structures that influence their

13 trafficking and intracellular distributions, but how the cell discerns among the different ceramides to

14 affect such sorting remains unknown. To address mechanism, we synthesized a library of GM1

15 glycosphingolipids with naturally varied acyl chains and quantitatively assessed their sorting among

16 different endocytic pathways. We found that a stretch of at least 14 saturated carbons extending from

$17 \mathrm{C} 1$ at the water-bilayer interface dictated lysosomal sorting by exclusion from endosome sorting

18 tubules. Sorting to the lysosome by the C14*-motif was cholesterol dependent. Perturbations of the

$19 \mathrm{C} 14 *$-motif by unsaturation enabled GM1 entry into endosomal sorting tubules of the recycling and

20 retrograde pathways independently of cholesterol. Unsaturation occurring beyond the C14*-motif in

21 very long acyl chains rescued lysosomal sorting. These results define a structural motif underlying

22 membrane organization of sphingolipids and implicate cholesterol-sphingolipid nanodomain formation

23 in sorting mechanisms. 
Introduction

Membrane function depends on the structural variations and dynamic physical properties of the acyl chains that form each class of its lipid components. But how the acyl chains operate to differentially affect the membrane biology of a lipid, and why so many different acyl chain structures are conserved within each lipid species remain in large part unexplained.

The complex sphingolipids, comprised of the glycosphingolipids (GSLs) and sphingomyelin, constitute 10-20 mole \% of plasma membrane lipids $(1,2)$, and they provide a tractable experimental system to examine how acyl chain structure affects lipid function. In many cases,

33 the activities of complex glycosphingolipids appear to depend upon locally segregating into 34 liquid-liquid phase-separated nanodomains that form upon close interactions with cholesterol 35 (3-6), the cortical cytoskeleton (7-11), and other scaffolding proteins (12-14). This is the lipidraft or lipid nanodomain hypothesis (15-20). Considerable evidence shows that nanodomains

37 are critical for key cellular processes including membrane protein trafficking, signal transduction, and receptor cell surface distributions $(16,21-27)$. Because of their functions in vesicular trafficking, some GSLs forming membrane nanodomains are co-opted by viruses and toxins, and by endogenous lectins, as platforms for entry into the cell and causation of disease

41 (28-34).

42 Here, we studied ganglioside GM1, the glycosphingolipid receptor that mediates cholera toxin

43 (CTx) entry into host cells. GM1 typifies the complex sphingolipids in basic structure and

44 function. The different GM1 species, like all other complex sphingolipids, are comprised of a 45 hydrophilic extracellular moiety (a specific pentasaccharide for GM1, one or more sugars for 46 the other GSLs, and phosphocholine for sphingomyelin) coupled to a ceramide that anchors the 47 molecule in the membrane. The ceramide moieties consist of a sphingosine base 48 (predominantly 18 hydrocarbons long, $\mathrm{C} 18: 1^{\Delta 4}$, trans) linked to a fatty acid by an amide bond to 49 the $\mathrm{C} 1$ carbon positioned at the lipid bilayer-water interface. The sphingosine base varies in 50 structure only to a small degree, but the acyl chain can display considerable heterogeneity in 51 both length (C14 to C26) and saturation (single cis or no double bond) $(35,36)$. Because these 52 lipids contain large hydrophilic extracellular head groups, they are trapped in the outer leaflet 
53 of the membrane bilayer and their distribution throughout the cell occurs only by vesicular 54 traffic $(37,38)$. This can be quantitatively measured and compared for GM1 species with 55 identical head groups but with varied acyl chains in their ceramide moieties.

56 We previously found the different acyl chains in the ceramide moiety of GM1 act decisively in

57 trafficking the toxin into the different endocytic and sub-cellular compartments required for the 58 induction of toxicity $(39,40)$. GM1 species containing ceramides with "kinked" shapes caused 59 by a cis-double bond in the acyl chains $\left(C 16: 1^{\Delta 9}\right.$ or $\left.C 18: 1^{\Delta 9}\right)$ sorted into the recycling and retrograde endosome pathways, and GM1 species containing ceramides with saturated acyl

61 chains of the same length, C16:0 and C18:0, but with "straight conical" shapes, sorted to the 62 lysosome instead (40). Additional evidence that acyl chain structure of the complex 63 sphingolipids may affect trafficking was recently shown for ceramide-anchored proteins in the 64 biosynthetic pathway of yeast (41). But how the cell discerns among the different ceramide 65 moieties to affect this acyl chain-based sorting has not been explained.

To address these problems, we synthesized a library of eleven GM1 species with ceramides

67 reflecting the naturally occurring variations of fatty acyl chain length and saturation. This provided a direct test of GSL structural biology in ways not previously possible.

69 In mammals, the single cis-double bond in the acyl chain of the unsaturated ceramide is created by palmityl/stearoyl-CoA desaturase (SCD). The enzyme installs a cis-double bond invariably at

71 the $\Delta^{9}$-position of $\mathrm{C} 16: 0$ or C18:0 fatty acids, creating a 'kinked' shaped lipid (42). When 72 coupled to sphingosine by the ceramide synthases, and then incorporated into the complex 73 sphingolipids, the kink in the acyl chain of these ceramide species is positioned where it should 74 impair close packing against the rigid planar surface of cholesterol (43). On the other hand, GSL 75 species containing ceramides with longer unsaturated acyl chains will have the cis-double 76 bonds placed deeper within the membrane bilayer. They are built upon the same C18:1 acyl 77 chains generated by the $\Delta 9$-desaturase, but lengthened at the carboxy-terminus by the ELOVL 78 elongases (44). Consequently these unsaturated very long acyl chains contain longer stretches 79 of saturated hydrocarbons extending from the water-bilayer interface into the membrane 80 bilayer. In some cases, the cis-double bond will be positioned beyond the planar surface of 
81 cholesterol. We reasoned that these unsaturated, and "kinked", very long acyl chain GSLs might

82 still assemble closely with cholesterol to form nanodomains and function accordingly - thus

83 providing a structural test of sphingolipid behavior in live cells.

84 Our results define a structural motif in the ceramide acyl chain of the GSLs that dictates

85 endosomal sorting and with broad implications for current models of membrane structure and

86 function.

87 


\section{Results}

89 The eleven GM1 molecules synthesized for testing contained saturated and mono-unsaturated 90 acyl chains (Fig. 1B) produced naturally in mammalian cells $(45,46)$. Three additional molecules,

91 the $\mathrm{GM} 1 \mathrm{C} 12: 1^{\Delta 6}, \mathrm{C} 12: 0$, and $\mathrm{C} 16: 1^{\Delta 9}$ species, were prepared to contain non-native ceramide

92 acyl chains that broadened the testing of the hypothesis (Fig. 1B, Suppl. Fig. 1A). The cellular

93 and membrane behaviors of each GM1 species were studied by coupling the extracellular

94 oligosaccharide to a 'reporter'-peptide containing biotin and fluorophore (Suppl. Fig. 1A), or 95 directly to a fluorophore $(40,47,48)$.

96 As a first test of membrane behavior, the liquid ordered $\left(L_{o}\right)$ and liquid disordered $\left(L_{d}\right)$ phase 97 preferences for each of the GM1 species were defined in vitro using giant plasma membrane 98 vesicles (GPMVs) prepared from different cell lines as indicated (Suppl. Figs. 1B - D). Certain 99 saturated lipids and sterols can condense into higher ordered assemblies within membranes, 100 termed the $L_{o}$ phase. This separates them from the other lipids present in the same membrane 101 termed the $L_{d}$ phase. Phase partitioning into the $L_{o}$ phase can be considered a feature of 102 nanodomains in live cells $(49,50)$. We found the major determinant for the phase behaviors of 103 the different GM1 species in GMPVs to be the presence or absence of a cis-double bond in the 104 ceramide acyl chain. The unsaturated GM1 species segregated into the $L_{d}$ phase irrespective of 105 the cell type-specific GMPV tested. GM1 with fully saturated ceramide acyl chains associated 106 exclusively with the $L_{\circ}$ phase. Thus, partitioning into the $L_{o}$ phase in GPMVs depended on full 107 saturation of the fatty acyl chain.

108 A saturated acyl chain length of at least 14 carbon atoms in the GM1 ceramide directs sorting to the lysosome

110 We next used lattice light-sheet and airyscan microscopy to image the different fluorescently111 labeled GM1 species in the sorting endosome and their ability to enter endosomal sorting 112 tubules. Endosomal sorting tubules affect the trafficking of membrane and cargo away from the 113 lysosome and into the recycling pathway for return back to the plasma membrane (PM), or into 114 the retrograde pathway for transport to Golgi and endoplasmic reticulum (ER) (schematic, Fig. 
115 1A). For these studies, human A431 epithelial cells were co-incubated with nM concentrations 116 of the different GM1 species together with transferrin (Tfn) and dextran. Tfn and dextran were 117 used to mark the endosome recycling and the lysosome pathways respectively $(51,52)$. Sorting 118 endosomes, which serve all pathways, were operationally defined as Tfn and dextran double 119 positive vesicles, and sorting tubules emerging from these structures were identified by their 120 Tfn-only content. We tested several cell lines for this assay. The A431 cell line selected for these 121 studies exhibited highly elongated and easily visualized tubules emerging from the sorting 122 endosome. The same basic phenotypes, however, were observed in other A431 clones, and 123 HeLa (Suppl. Fig 1D and E), MDCK II, Caco2, and HT29 cell lines which all lacked the unusually 124 elongated tubules (data not shown).

125 We found that the GM1 species with ceramides containing short saturated acyl chains $(\leq \mathrm{C} 14: 0)$ 126 or unsaturated acyl chains $\mathrm{C} 12: 1^{\Delta 6}$ through $\mathrm{C} 22: 1^{\Delta 13}$ efficiently co-localized with Tfn in 127 endosome sorting tubules (Fig. 1C, left panels and quantified in Fig. 1E and Suppl. Video 1). The 128 GM1 species with ceramides containing long saturated acyl chains $\geq$ C16:0, however, did not 129 (Fig. 1C, right panels, Fig. 1E and Suppl. Video 2). They were localized only to the body of the 130 sorting endosome. We tested this observation again using the C18:0 and C18:1 ceramide pair 131 loaded together into the same cells and obtained the same results (Fig. 1D and 1F and Suppl. 132 Video 2).

133 Notably, we also found that the GM1 species containing ceramides with unsaturated but very 134 long acyl chains ( $\geq C 24: 1^{\Delta 15}$ in length) were localized only in the body of the sorting endosome, 135 behaving like the GM1 species with fully saturated acyl chains. These GSLs did not enter the 136 narrow highly-curved sorting tubules despite the presence of a cis-double bond in their 137 ceramide acyl chain (Fig. 1B, 1C lower right panels, and Fig. 1E). Additionally, the GM1 species 138 with $\mathrm{C} 14: 0$ or $\mathrm{C} 22: 1^{\Delta 13}$ fatty acids displayed intermediate and closely similar phenotypes. They 139 still entered endosome sorting tubules but less efficiently (measured as lower fluorescence 140 intensity in recycling endosomal tubules) (Fig. $1 \mathrm{G}$ and $\mathrm{H}$ left panel). This observation was not 141 explained by reduced endocytic uptake of the lipids (Fig. $1 \mathrm{H}$ right panel). Closely similar 142 phenotypes for all the GM1 species tested were found in Hela cells (Suppl. Fig. 1E and F), and 
143 for the other epithelial cell lines noted above (data not shown). Thus, while the presence or

144 absence of a cis-double bond in the ceramide acyl chain dictated $L_{o}$ and $L_{d}$ phase partitioning in

145 GMPVs, the position of the double bond within the acyl chain acted as the decisive factor for 146 endosomal sorting in live cells. Lipid shape, on its own, was not sufficient to explain the

147 differential sorting observed. These results, especially considering the intermediate behaviors 148 of the $\mathrm{C} 14: 0$ and $\mathrm{C} 22: 1^{\Delta 13}$ ceramide species, showed that a saturated acyl chain length of at 149 least 14 carbon atoms plus one, extending from the C1 amide bond at the water-bilayer 150 interface, was necessary for retention of GM1 in the body of the endosome. We henceforth 151 name this structure the C14*-motif (Fig. 1G).

\section{Independence from Lectin binding}

153 The C14*-motif may be necessary but not sufficient to explain the differential endosomal 154 sorting of GM1 as endogenous lectins capable of scaffolding the lipid may be involved (34). 155 Such an effect on GM1 trafficking is perhaps best typified by multi-valent binding to CTxB (33, $15640,53-55)$. As such, we first tested if binding CTxB might act on the different GM1 species to 157 affect changes in their $L_{d}$ and $L_{o}$ phase behaviors as assessed in GMPVs. The addition of CTxB 158 caused a switch from $L_{d}$ to $L_{o}$ phase preference, but only for the GM1 species with very long 159 unsaturated acyl chains $\geq 22: 1^{\Delta 13}$ (Suppl. Fig.1G). The phase preferences for the other GM1 160 species were not affected.

161 To test if in live cells cross-linking GM1 by endogenous lectins may be required for its 162 endosomal sorting, we studied the GM1 C16:0 and C16:1 ${ }^{\Delta 9}$ pair, and GM1 C24:1 ${ }^{\Delta 15}$. Here, 163 intracellular trafficking of the different GM1 species was studied in A431 cells continuously 164 exposed to $100 \mathrm{mM}$ lactose or sucrose to compete against binding the GM1 oligosaccharide 165 moiety by endogenous lectins (56). We found no effect of the disaccharide treatments (Suppl. 166 Fig. $1 \mathrm{H})$. Control studies showed that $100 \mathrm{mM}$ lactose was sufficient to block the high affinity 167 binding of CTXB to GM1 on the A431 cell surface (Suppl. Fig.1I). Thus, binding by endogenous 168 galactose- or glucose-binding lectins does not appear to be required for the observed GM1 endosomal sorting (Fig. 1B) - in these cases, the C14*-motif is both necessary and sufficient. 


\section{Differential sorting between the lysosomal and recycling pathways}

171 To quantitatively test if endosomal GM1 sorting is based on the C14* motif, we developed a

172 FACS based assay to measure GM1 sorting between recycling endosomes and lysosomes (Fig.

$1731 \mathrm{~A}$, Pathways A vs C). For these studies, we synthesized the GM1 library coupled to the all-D

174 amino acid 'reporter'-peptide containing only a biotin functional group (Suppl. Fig. 1A).

175 Conditions for incorporating the different GM1 species into the PM at similar amounts were

176 determined experimentally (Fig. 2A, left panel). After a 3h 'chase', the amount of the biotin-

177 labeled-GM1 located at the cell surface was determined by FACS measured by binding of

178 fluorescently-labeled streptavidin (Fig. 2A and 2B). We found that the GM1 species which 179 entered endosome sorting tubules and lacked the C14* motif (GM1 C12:0, C14:0, C16:1 ${ }^{\Delta 9}$, $180 \mathrm{C} 18: 1^{\Delta 9}$ and $\mathrm{C} 22: 1^{\Delta 13}$, Figs. $1 \mathrm{E}$ and $2 \mathrm{~A}$ and $\mathrm{B}$ magenta points) were recycled after endocytosis. 181 They were detected on the PM at significantly higher levels compared to the GM1 species 182 containing the $\mathrm{C} 14^{*}$-motif (GM1 C16:0, C18:0, C24:1 ${ }^{\Delta 15}, \mathrm{C} 24: 0$ and $\mathrm{C} 26: 1^{\Delta 17}$, Fig. 2A and 2B 183 turquoise points). Even after longer chase times (> $6 \mathrm{~h}$ ), the GM1 C16:1 ${ }^{\Delta 9}$ species still localized 184 to the PM and within endosomal recycling tubules containing Tfn; while the GM1 C16:0 species 185 localized strictly to intracellular vesicles stained by lysotracker ${ }^{\mathrm{TM}}$ (marking late endosomes and 186 lysosomes, Suppl. Fig. 2A). We observed no confounding effects of GM1 treatment on 187 endocytosis per se - as measured by dextran (fluid-phase) or Tfn (receptor-mediated) uptake 188 (Suppl. Fig. 2B). In addition, the results could not be simply explained by differential endocytic 189 uptake mechanisms into caveolae - as neither the $\mathrm{C} 18: 1^{\Delta 9}$ nor $\mathrm{C} 18: 0$ species were sensitive to 190 CRISPR-Cas9 deletion of cavin1 (Suppl. 2C). Thus, a saturated acyl chain length of at least 14 191 saturated carbon atoms plus one (the C14*-motif) drives endosomal sorting of GM1 to the 192 Iysosome, with GM1 C14:0 and C22:1 ${ }^{\Delta 13}$ displaying intermediate phenotypes (Fig. 1A pathway 193 C, and Fig. 2C). We note again that lysosomal sorting was rescued for the unsaturated GM1 194 species containing very long unsaturated acyl chains (i.e. unsaturated "kinked shaped" fatty 195 acids containing the C14*-motif"). 
197 The nanodomain (lipid raft) hypothesis posits strong dependence on close assembly between

198 GSLs and membrane cholesterol. We reasoned, however, that such cholesterol dependence

199 should be evident for endosomal sorting of only the GM1 species with ceramide structures

200 containing the $\mathrm{C} 14^{*}$-motif - if the motif acts as we hypothesize by allowing close alignment

201 against the rigid and flat surface of cholesterol $(3,6,43)$. Likewise, endosomal sorting of the

202 GM1 species with cis-double bonds at positions disrupting the C14*-motif should behave

203 independently of membrane cholesterol. But the GM1 species with a cis-double bond located

204 beyond the C14*-motif, as seen in the naturally occurring very long acyl chains (> C22:1 $1^{\Delta 13}$ acyl

205 chains), should again show cholesterol-dependence.

206 To test this idea, we studied the endosome recycling of the different GM1 species in A431 cells

207 mildly reduced of membrane cholesterol: either by treatment with low doses of the small

208 molecule inhibitors of cholesterol metabolism simvastatin, T0901317, or U18666A; or as we

209 found experimentally by CRISPR-Cas9 knock out of serine-palmityltransferase-2 (SPTLC-2),

210 ceramide synthase 5 (CerS5), or ceramide synthase 2 (CerS2), or by simply growing the cells in

211 over-confluent conditions. In all cases, the experimental conditions were found to lower plasma

212 membrane cholesterol content as measured by loss of filipin staining (Suppl. Fig. 2D, left panel),

213 and further evidenced by increase in the general polarization (GP) of laurdan as assessed in

214 GPMVs (implicating increased membrane fluidity typifying cholesterol depleted membranes,

215 Suppl. Fig. 2D, right panel). We also conducted lipidomic profiling by mass spectrometry, which

216 showed in all cases that cells depleted of cholesterol had only minor changes in the different

217 species of the major membrane lipid classes: phosphatidylethanolamine (PE),

218 phosphatidylglycerol (PG), and phosphatidylcholine (PC) (Suppl. Fig. 3D). Additionally, and in all

219 cases, cells depleted in cholesterol by our methods retained their normal endosomal and

220 overall morphologies and endocytic uptake rates with only minor variations (Fig. 2E left panel

221 and Suppl. Fig. 2E). We also note that cholesterol depletion induced by over-confluent cell

222 culture, the least invasive perturbation, had only minimal effects on the overall lipidome and no

223 detectable changes on phase preferences for any of the different GM1 species in GPMVs

224 (Suppl. Fig. 2H). 
225 Consistently, for all methods, we found no effect of cholesterol depletion on the recycling of

226 GM1 species lacking the C14*-motif (Fig. 2D magenta points, and Suppl. Fig. 2F, left table). In

227 contrast, cholesterol depletion uniformly affected the late endosome/lysosome sorting of GM1

228 species with ceramide moieties containing the $\mathrm{C} 14^{*}$-motif. This included the saturated acyl

229 chains $\geq$ C16:0 and notably the unsaturated ceramides with very long unsaturated acyl chains $\geq$

$230 \mathrm{C} 24: 1^{\Delta 15}$. In cholesterol depleted cells, these GM1 species were no longer strictly sorted into

231 the late endosome/lysosome - instead, large fractions of these lipids were recycled back to the

232 PM (Fig. 2D turquoise points and Suppl. Fig. 2F, right table) and co-localized within Tfn-

233 containing endosome recycling tubules (where none was found before) (Fig. 2F and G). The

234 effect of cholesterol depletion on lysosomal sorting was specific for GM1, as lysosomal sorting

235 for the epidermal growth factor (EGF) receptor or the low density lipoprotein receptor LDL-R

236 were not affected (Fig. 2E right panel and Suppl. Fig. 2G). Thus, the sorting step for trafficking

237 GM1 into the lysosome appears to be dependent on membrane cholesterol, but only for the

238 GM1 species with ceramide structures containing the C14*-motif and predicted to interface

239 tightly alongside the sterol - consistent with the nanodomain hypothesis.

Sorting into retrograde and recycling pathways by bulk membrane flow

241 We next considered the possibility that the GM1 species lacking the C14*-motif may enter 242 endosome sorting tubules by default, following bulk membrane flow (57). If this were the case, 243 we reasoned these GM1 species should enter all pathways emerging from the sorting 244 endosome, including the retrograde pathway to the trans Golgi and ER. For these studies, we

245 used the B-subunit of cholera toxin (CTxB). Though CTxB scaffolds GM1 into a multimeric 246 complex affecting its membrane behavior $(54,58,59)$, ceramide structure still plays a decisive 247 role in endosomal sorting into the ER (40) and in liquid phase partitioning in GMPVs (Suppl. Fig.

248 1G). For these studies, the different GM1 species were applied to a HeLa cell line that 249 endogenously lacks GM1. CTxB trafficking into the ER was quantitatively measured using a 250 FACS-based split-GFP assay recently developed by our group (60). We found that only the short 251 chain $(\leq \mathrm{C} 14: 0)$ or unsaturated GM1 species $\left(\leq \mathrm{C} 22: 1^{\Delta 13}\right)$, both lacking the $\mathrm{C} 14^{*}$-motif, efficiently 252 trafficked CTxB into the ER (Fig. 3A and 3B). The GM1 species containing the C14*-motif did 
not. This includes the GM1 species with ceramide structures containing long saturated acyl chains ( $\geq$ C16:0), and those containing unsaturated but very long acyl chains $\left(\geq C 24: 1^{\Delta 15}\right)$, which rescues the $\mathrm{C} 14 *$-motif and its function. Thus, the requirement for acyl chain structure directing the different GM1 species into the retrograde endosomal pathway phenocopied exactly the structures required for sorting into the recycling pathway.

258 To confirm these results, we took an orthogonal approach and generated A431 cells containing 259 CRISPR-Cas9 deletion of CerS2, the ceramide synthase producing very long acyl chain ceramides 260 (> C20), or deletion of CerS5, the synthase producing ceramides containing short acyl chain 261 ceramides ( $\leq \mathrm{C} 16)(61)$. These deletions genetically bias the structures of the endogenous GM1 262 ceramide moieties in CerS2 and CerS5 KO cells as just described. The approach was validated by 263 lipidomic profiling of sphingomyelin (SM). SM and GSLs share the same ceramide biosynthetic 264 pathway and the abundance of SM in cell membranes allowed for the most conclusive analysis 265 of endogenous ceramide structures. In wild type A431 cells, the major SM species contained 266 C18:0 (45\%), followed by C24:1 (15-18\%), and C18:1 (10\%) acyl chains (Suppl. Fig. 3C). 267 Sphingomyelin containing ceramides with short acyl chains $\leq$ C16 (6-8\%) were also reproducibly 268 detected ( $n=8$ replicates). As expected in cells lacking CerS2, the SM species most strongly 269 depleted were ceramides containing the very long acyl chains, but with concomitant increase in 270 C18:0 and C18:1; while in cells lacking CerS5, the SM species most strongly depleted were the 271 short acyl chains $\leq$ C16, and unexpectedly the C18 acyl chain species, with increases in C22:0 272 and $\mathrm{C} 24: 1$.

273 When tested for GM1-dependent trafficking of CTxB, we found that the retrograde pathway 274 was significantly reduced in those cells lacking CerS5 (Fig. 3C), consistent with 1) the loss of the 275 short and C18:1 acyl chain ceramides that lack the C14*-motif; and 2) the increased expression 276 of the very long chain saturated ceramide species C22:0 - including notably again those with an 277 unsaturated bond positioned more deeply in the membrane (GM1 C24:1 $\left.{ }^{\Delta 15}\right)$ - which contain the 278 C14*-motif. We found a slight increase in retrograde trafficking for cells depleted in Cer2, 279 consistent with depletion of only the very long chain ceramides and the modest increase in 280 ceramides with $\mathrm{C} 18: 1^{\Delta 9}$ (Fig. $3 \mathrm{C}$ and Suppl. Fig. 3C). Again, control studies showed that 
281 endocytosis and EGF/LDL-R trafficking to the lysosome in CerS5 and CerS2 KO cells were not 282 altered, or slightly enhanced (Fig. 2E, Suppl. Fig. 2G).

283 Opposite results were found for sorting CTxB to the acidic lysosome as predicted. This was 284 measured by FACS using CTXB coupled to the $\mathrm{pH}$-sensitive fluorophore pHrodo ${ }^{\text {TM }}$. We found 285 that CTxB-pHrodo ${ }^{\mathrm{TM}}$ fluorescence was reduced in the A431 cells deleted in CerS2, consistent 286 with loss of very long acyl chain ceramides containing the C14*-motif and increase in the 287 unsaturated $\mathrm{C} 18: 1^{\Delta 9}$ ceramide lacking the motif (Fig. 3D). For cells deleted in CerS5, which 288 expressed higher levels of very long acyl chain ceramides $\left(C 20: 0, C 22: 0\right.$, and $\left.C 24: 1^{\Delta 15}\right)$, we found 289 a slight increase in lysosomal transport - consistent with the enhanced expression of GM1 lipids 290 containing the $\mathrm{C} 14^{*}$-motif. Thus, the genetically modified cell lines phenocopied the trafficking 291 of GM1 observed using exogenously applied and structurally defined GM1 species.

292 Cholesterol depletion had the same effect (Fig. 3E) - here we also observed an increase in 293 retrograde trafficking to the ER (Fig. 3F). These results implicate a selective and cholesterol 294 dependent process for retention within the body of the endosome followed by maturation to 295 lysosomes for the GM1 ceramides that contain the C14*-motif (Fig. 3E and 3F). GM1 species 296 lacking the $\mathrm{C} 14^{*}$-motif escape this lysosomal sorting step and sort, perhaps passively by 297 following bulk membrane flow, into endosome tubules serving the recycling and retrograde 298 endocytic pathways.

299 The C14* motif drives nanodomain assembly and segregation at the plasma membrane

300 The cholesterol dependency observed for C14*-motif based GM1 sorting to the lysosome is 301 consistent with the idea that the mechanism of such sorting may involve assembly into 302 membrane nanodomains. To test this, we imaged the GM1 C18:0 and C18:1 ${ }^{\Delta 9}$ species in sorting 303 endosomes artificially enlarged to enable visualization by over expression of dominant active 304 Rab5Q79L (tagged with EGFP). We found clear separation of the two GM1 species in the plane 305 of the endosomal membrane (Fig. 4A and B), and clear segregation between GM1 C18:0 (but 306 not GM1 C18:1 ${ }^{\Delta 9}$ ) and the Tfn-Tfn-R complex. Again, the unsaturated very long acyl chain GM1 $307 \mathrm{C} 24: 1^{\Delta 15}$, which also contains the C14*-motif, was found to behave like GM1 C18:0. These 
results imply nanodomain formation within the sorting endosome. Notably, the clear segregation of the two unsaturated GM1 species $C 24: 1^{\Delta 15}$ and $C 18: 1^{\Delta 9}$ into distinct endosomal nanodomains in live cells was not predicted by their phase behaviors in GMPVs (Suppl. Fig. 1B -

311 D). In those studies, phase separation was dictated by the presence or absence of cis-double 312 bond (kinked or straight shapes). In live cells, as evidenced here, we find the C14*-motif to be 313 the decisive factor - not lipid shape.

314 We were then curious to know if such segregation by nanodomain formation may also occur in 315 the plasma membrane, before the lipids were internalized by endocytosis. To test this, we 316 measured the diffusion rate of GM1 C18:0, C18:1 ${ }^{\Delta 9}$, and $\mathrm{C} 24: 1^{\Delta 15}$ in plasma membranes of A431 317 cells using FRAP. We found slower rates of fluorescence recovery (FRAP) for the GM1 C18:0 and $318 \mathrm{C} 24: 1^{\Delta 15}$ species (which contain the C14*-motif) compared to GM1 C18:1 ${ }^{\Delta 9}$ species (which lacks 319 the C14*-motif) (Fig. 4C). Thus, even in the plasma membrane, the distinct behaviors among 320 the different GM1 species appears to be dictated by the C14*-motif. The slower diffusion of the 321 GM1 C18:0 and C24:1 $1^{\Delta 15}$ species is consistent with their assembly in membrane nanodomains 322 linked to the subcortical cytoskeleton (62).

323 To test if these differences in plasma membrane mobility correlated with differences in uptake, 324 we measured the apparent rate of endocytosis. Here, we used SVGA cells endogenously 325 expressing Rab5-GFP to measure the co-localization between the different GM1 species and 326 Rab5 early endosomes. Uniformly, after short 5 to 10 min incubations, little co-localization with 327 Rab5-endosomes was observed for GM1 ceramides containing fully saturated C16:0, C18:0, and 328 C24:0 acyl chains and for the very long unsaturated acyl chain GM1 C24:1 ${ }^{\Delta 15}$ (Fig. 4D) These 329 ceramides all contain the C14*-motif. The result implicates slow rates of endocytosis, or very 330 fast transition to Rab 7 late endosomes (or both) for these GM1 species. In contrast, we found a 331 much higher degree of co-localization between the Rab5-endosome and GM1 ceramides 332 containing the $\mathrm{C} 16: 1^{\Delta 9}$ and $\mathrm{C} 18: 1^{\Delta 9}$ acyl chains, which lack the C14*-motif - consistent with 333 rapid rates of endocytosis, or efficient recycling (or both). The same results were obtained using 334 Tfn, which we used as a control, phenocopying the $C 16: 1^{\Delta 9}$ and $C 18: 1^{\Delta 9} \mathrm{GM} 1$ species (Fig. 4D). 
When the SVGA cells were depleted of cholesterol, however, co-localizations of the GM1 C16:0 and very long C24:1 $1^{\Delta 15}$ acyl chain species with Rab5 endosomes were increased (Fig. 4E). These lipids also displayed an increase in their PM mobility after cholesterol depletion as assessed by FRAP (Fig 4F, compare black with magenta lines and Suppl. Fig. 4A for quantification). When the SVGA cells were supplemented with additional exogenous cholesterol, however, the degree of colocalization with Rab5 endosomes was diminished for the same GM1 C16:0 and C18:0 species, and for the very long unsaturated acyl chain $\mathrm{C} 24: 1^{\Delta 15}$ species - compared to control untreated cells (Fig. 4G and Suppl. Fig. 4B). This phenotype induced by cholesterol addition was more readily apparent in HT29 cells, where PM localization was prominently marked at cell-cell contact sites. Here, these lipids appeared to be visibly "trapped" on the PM after cholesterol addition (Fig. 4G and H, and Suppl. Fig. 4D). Notably, the addition of cholesterol to SVGA cells had no detectable effect on the apparent efficiency of co-localization with Rab5-endosomes for the unsaturated GM1 species $\mathrm{C} 16: 1^{\Delta 9}$ and $\mathrm{C} 18: 1^{\Delta 9}$ (all lacking the $\mathrm{C} 14^{*}$-motif) or for Tfn used as control. And we found no confounding effect of cholesterol treatment on fluid-phase dextran endocytic uptake or EGF-R/LDL-R sorting to the lysosome (Suppl. Fig. 4F).

Further evidence that excess membrane cholesterol caused retention of GM1 species containing the $\mathrm{C} 14^{*}$-motif on the PM was obtained in A431 cells as assessed by FACS (Suppl. Fig. 4E) and by confocal microscopy (Fig. 4H). - and with no evidence for co-localization within

353 Tfn-positive endosome recycling tubules (Suppl. Fig. 4D). We also found a striking reduction in 354 PM diffusion rates for the $\mathrm{C} 18: 0$ and $\mathrm{C} 24: 1^{\Delta 15}$ species in A431 cells treated with exogenous 355 cholesterol - as assessed by FRAP (Fig. $4 \mathrm{I}$ and Suppl. Fig. 4A and see (63)). These results suggest nanodomain assembly for the C16:0, C18:0, and notably C24:1 ${ }^{\Delta 15} \mathrm{GM} 1$ lipids.

357 Thus overall, we found that the different GM1 species are sorted within live cells based on the

358 C14*-motif. These GM1 species were retained within the body of the sorting endosome for 359 maturation along the lysosomal pathway. And they exhibited dependence on membrane 360 cholesterol for their sorting and biophysical behaviors, consistent with an affinity for assembly 361 into membrane nanodomains (Fig. 5). In contrast, the GM1 species containing mono362 unsaturated acyl chains of 12 to 22 hydrocarbons atoms in length (thus lacking the C14*-motif) 
bioRxiv preprint doi: https://doi.org/10.1101/2021.04.26.440603; this version posted April 26, 2021. The copyright holder for this preprint (which was not certified by peer review) is the author/funder, who has granted bioRxiv a license to display the preprint in perpetuity. It is made available under aCC-BY-NC-ND 4.0 International license.

363 did not display dependence on cholesterol for their membrane behaviors. And unlike the fully

364 saturated GM1 species, these GSLs entered endosome sorting tubules of the recycling and

365 retrograde endosome pathways.

366 


\section{Discussion}

368 The results of our studies show that mammalian cells sort GM1 sphingolipids between 369 endosomes and endosome sorting tubules, and within the plane of the PM, based on their 370 ceramide acyl chain structure. This is explained by differential association with membrane 371 cholesterol - likely mediated by lipid nanodomain formation (Fig. 2C and schematic in Fig.5). 372 Lipid shape on its own $(55,64-66)$ cannot fully explain the selective entry of the different GM1 373 species into the narrow highly curved endosome sorting tubules, or their retention in the body 374 of the sorting endosome. The differential sorting phenotypes observed were strictly dependent 375 on membrane cholesterol content and ceramide structure, and the same structure-function 376 relationships operated across multiple cell types known to vary in their overall membrane 377 thickness, fluidity, and cholesterol content (our unpublished data and (67-69)). These results 378 define a structural organizing principle for complex sphingolipid behavior in native 379 heterogeneous and dynamic live cell membranes. The complex sphingolipids critically require 380 more than 14 saturated hydrocarbons in the ceramide acyl chain extending from the C1-amide 381 at the water-bilayer interface for optimal association with cholesterol. And this decisively 382 affects their direction of endosome sorting. GSL species lacking these features escape the 383 apparently active lysosomal sorting step and enter endosome sorting tubules of the recycling 384 and retrograde pathways instead. These observations experimentally verify in live cells one of 385 the underlying and fundamental biophysical processes hypothesized to shape lipid trafficking, 386 membrane architecture, and cell function. It is possible the C14*-motif may also explain the 387 differential sorting of ceramide-linked proteins in the secretory pathway recently observed in 388 yeast (41).

389 In nature, the cis-double bond in the GM1 species containing mono-unsaturated acyl chains of 39016 to 22 hydrocarbons atoms in length is positioned at carbon atom 9 or $13\left(\Delta^{9}\right.$ and $\left.\Delta^{13}\right)$ 391 extending from the amide linkage to sphingosine, and thus located within the outer leaflet of 392 the membrane bilayer. Within this area, the "kink" in the acyl chain caused by unsaturation 393 interferes with alignment against the flat and rigid sterol structure of cholesterol $(43,70-74)$. 394 These GM1 species entered endosome sorting tubules of the recycling and retrograde 
endosome pathways, and their trafficking was not dependent of cholesterol. On the other hand, the GM1 species containing the very long mono-unsaturated acyl chains $\left(\mathrm{C} 24: 1^{\Delta 15}\right.$ and $\mathrm{C} 26: 1^{\Delta 17}$ ) trafficked to the lysosome - phenocopying the fully saturated GM1 species even though they also contained a cis-double bond "kink" in their ceramide. Here, the structural analysis was most informative. The cis-double bond in these native acyl chains is located at $\Delta^{15}$ or $\Delta^{17}$, below the stretch of 14 saturated hydrocarbons from the amide linkage at the membrane surface $(44,70)$, and below the expected interface with the sterol rings of cholesterol (43). Thus, the acyl chains of these GM1 species recapitulated exactly the minimal saturated hydrocarbon chain length that we found required for endosome sorting of the GSLs to the lysosome. Notably, the membrane behavior of these very long unsaturated GM1 species

405 in live cells was strictly dependent on membrane cholesterol, analogous to the fully saturated 406 acyl chain GM1 species. Two species GM1 C14:0 and GM1 C22:1 ${ }^{\Delta 13}$ had intermediate 407 phenotypes for cholesterol dependence - these ceramides have acyl chain lengths that border 408 on the minimal $\mathrm{C} 14^{*}$-motif required for functional association with cholesterol.

409 The strong dependence on cholesterol for the membrane behaviors of the saturated and very 410 long chain unsaturated GM1 species conforms to central tenants of the lipid raft hypothesis.

411 The raft hypothesis posits that certain lipids and cholesterol phase separate into ordered and 412 tightly packed domains, mediated through hydrogen bonding and van der Waals forces, and 413 shaped by cellular protein scaffolds, including by bonding to the cortical actin cytoskeleton (18, $41420,75)$. For the saturated and very long chain unsaturated GSLs, we find evidence for their 415 assembly into membrane nanodomains: perhaps best evidenced by the segregation of these 416 GM1 species in separate regions of artificially enlarged early endosomes. Our results are 417 consistent with another structural factor implicated in nanodomain assembly for GPI-anchored 418 proteins and recently for $\operatorname{GM1}$ ((76) and our unpublished results, Arumugam et al.): the need 419 for direct extension of outer membrane lipids into the inner-membrane lipid leaflet for 420 assembly with phosphatidyserine and the cortical actin cytoskeleton. In those studies, we found 421 that the ceramide $\mathrm{C} 14^{*}$-motif was required for features of nanodomains with linkage to the 422 cortical cytoskeleton as assessed by fluorescence correlation microscopy. Nanodomain 
423 formation, and GM1 trafficking, may also be affected by extracellular factors that induce lipid 424 clustering (14), as we found for CTxB (40).

425 The structural diversity of ceramide moieties comprising the complex sphingolipids that occur 426 in nature is perhaps best defined for sphingomyelin, which is the most highly prevalent complex 427 sphingolipid, and thus most easily and reproducibly studied. In our hands, and in the cell-type 428 examined, we found that the predominant ceramide moieties in sphingomyelin contained $429 \mathrm{C} 18: 0, \mathrm{C} 18: 1^{\Delta 9}, \mathrm{C} 24: 0$, and $\mathrm{C} 24: 1^{\Delta 15}$ acyl chains. This, and published lipidomic analyses of 430 sphingomyelin in other cell types, suggest the evolution of two 'bi-modal' structure-function 431 distributions for the complex sphingolipids: one between long and very long ceramide acyl 432 chains as proposed before [(46)61-64], and the other between saturation and unsaturation as 433 we find here. Experimentally, these two bi-modal distributions were evident in our structure434 function studies on GM1. In the case of unsaturation, the different membrane behaviors were 435 most readily apparent for the saturated GM1 C18:0 and the unsaturated C18:1 species. But this 436 dichotomy of saturation and unsaturation was not absolute - it also depended on acyl chain 437 length. In the case of GM1 species with very long C24:0, and C24:1 ${ }^{\Delta 15}$ acyl chains, for example, 438 these GSLs behaved mostly in the same way - even though one was saturated and the other 439 was not. Thus, acyl chain length in the native complex sphingolipids appears to over-ride the 440 functional impact of unsaturation, as long as the cis-double bond is positioned below a stretch 441 of saturated hydrocarbons of sufficient length (beyond the C14*-motif). The long and very long 442 acyl chains may also enable interdigitation with the contralateral lipid leaflet (77-79). Lipid 443 shape, on its own, does not appear to act decisively for entry of the GSLs into endosome sorting 444 tubules, or for their endosomal sorting - contrary to the impact of acyl chain unsaturation on $L_{d}$ 445 and $\mathrm{L}_{\mathrm{o}}$ phase separation and nanodomain assembly in GPMVs.

446 We also note that some very long unsaturated acyl chain GM1 species, typified by GM1 $447 \mathrm{C} 24: 1^{\Delta 15}$, exhibited graded membrane behaviors that bridged the dichotomy between 448 saturation and unsaturation seen in the GM1 C18:0 and C18:1 pair. These very long acyl chain 449 GSLs sorted predominantly to the lysosome, but a significant portion sorted also into the 450 recycling pathway - in contrast to their fully saturated counterparts. In addition, GM1 C24:1 ${ }^{\Delta 15}$ 
451 had a restricted but still higher degree of apparent endocytic uptake compared to its fully

452 saturated counterpart. Thus, the very long chain unsaturated GSLs appear to occupy a

453 functionally intermediate space. They may have been evolutionarily conserved according to

454 their graded membrane behaviors in membrane sorting so as to more readily enable

455 differential responses to small alterations in membrane structure and composition caused by

456 cell stress, cell differentiation, or even to the normal dynamic changes in membrane content

457 produced by trafficking from one organelle to another in cells at steady state (80).

458 In summary, our studies show that the membrane behaviors of the complex sphingolipids are

459 defined by two general rules of ceramide structure - hydrocarbon acyl chain length and 460 unsaturation. This includes the position of the cis-double bond with respect to the outer leaflet

461 of the membrane lipid bilayer and how acyl chain length and saturation affect the ability of

462 GSLs to associate with cholesterol and possibly to form nanodomains. These basic structural

463 principles are of general importance to cell and tissue biology, as the segregation and behavior

464 of lipids has fundamental physiological consequences for membrane organization and the 465 plasticity required to accommodate the diversity of membrane dynamics underlying cell 466 functions. 


\section{Material and Methods}

469

470

471

472

473

474

475

476

477

478

479

480

481

482

483

484

485

486

487

488

489

490

491

492

493

Dimethylformamide (DMF), dimethylsulfoxide (DMSO), methyl- $\beta$-cyclodextrin (M $\beta C D)$, soluble cholesterol complexed to $M \beta C D$, defatted bovine serum albumin (dfBSA), fetal bovine serum (FBS), fetal bovine serum depleted of HDL (HDL-), Simvastatin (statin), T0901317 (T09), U18666A (U18) and filipin III (filipin) were purchased from Sigma Aldrich (St. Louis, MO).

Dulbecco's modified Eagle's medium (DMEM) with or without phenol red, OptiMEM ${ }^{\circledR}$, Lipofectamine $^{\circledR}$ 2000, Laurdan (6-Dodecanoyl-2-Dimethylaminophthalene), FASTDil ${ }^{\mathrm{TM}} \mathrm{Dil}^{\Delta 9,12}$. $\mathrm{C}_{18}(3), \mathrm{ClO}_{4} \quad$ (1,1'-Dilinoleyl-3,3,3',3'-Tetramethylindocarbocyanine Perchlorate), FASTDio ${ }^{\mathrm{TM}}$ $\mathrm{DiO}^{\Delta 9,12}-\mathrm{C}_{18}(3), \mathrm{ClO}_{4}$ (3,3' Dilinoleyloxacarbocyanine Perchlorate)), various AlexaFluor ${ }^{\mathrm{TM}}$ (- 405, 488, - 568, or - $647 \mathrm{~nm}$ ) or pHrodo ${ }^{\mathrm{TM}}$ Fluor -NHS esters and -azide dyes, AlexaFluor ${ }^{\mathrm{TM}}$ and pHrodo $^{\text {TM} F l u o r ~ c o n j u g a t e s, ~ D e x t r a n ~ P a c i f i c ~ B l u e ~}{ }^{\mathrm{TM}}$ (3000 MW) and LysoTracker ${ }^{\mathrm{TM}}$ were purchased from Thermo Fisher Scientific (Waltham, MA).

\section{Cell culture}

Human epithelial A431, HT29, CaCo and Hela cells, as well as HEK293T cells were originally purchased from the American Tissue Culture Collection (ATCC, VA). Cells were maintained in Dulbecco's modified Eagle's medium (DMEM) containing 10\% FBS with penicillin and streptomycin. Cultures were split two days prior to experiment to achieve the desired confluency state. The CRISPR-Cas9 SVGA Rab5-GFP cell line was maintained in MEM containing $10 \%$ FBS.

For cholesterol addition and/or depletion assays, cells were cultured for $24 \mathrm{~h}$ in [50 - $100 \mathrm{nM}$ ] T0901317, or for $12 \mathrm{~h}$ in [1 $-2 \mu \mathrm{g} / \mathrm{ml}] \mathrm{U} 18666 \mathrm{~A}$ in full medium and for $24 \mathrm{~h}$ for [10 $\mu \mathrm{M}$ ] simvastatin in serum depleted of HDL. Soluble cholesterol complexed to M $\beta C D[120 \mu \mathrm{g} / \mathrm{ml}]$ was given in full media for 30 min or the indicated time. Efficacy of treatment was determined by fixing treated cells in $4 \%$ paraformaldehyde for $1 \mathrm{~h}$ and subsequently staining cells with [0.05 $\mathrm{mg} / \mathrm{ml}$ filipin III to determine plasma membrane cholesterol contents by fluorescence. Plasma membrane fluorescence intensity was measured using the Fiji (ImageJ, National Institutes of 
494 Health, Bethesda, MD, USA) segmented line tool to outline plasma membrane and mean grey 495 values were obtained.

496 To obtain enlarged Rab5 early endosomes, A431 cells were transfected using Lipofectamine ${ }^{\circledR}$ 4972000 and OptiMEM $^{\circledR}$ transfection method according to manufacturer's instructions and $500-$ $4981000 \mathrm{ng}$ of the Addgene plasmids (\#28046, \#35138).

\section{Generation of CRISPR-Cas9 KO cell lines}

500 CRISPR-Cas9 KO cell lines were generated using Addgene plasmid lentiCas9-Blast (\#52962) for 501 stable integration of Cas9 endonuclease and guide sequences were cloned into Addgene 502 plasmid lentiGuide-Puro (\#52963) against ceramide synthase 1, 2 and 5 (CerS1, 2 and 5), UDP503 glucosyl-ceramide glucosyltransferase (UGCG), and serine palmityl transferase 2 (SPTLC-2) (see

504 Table 1). Plasmids were verified by sequencing using IDT technologies (Coralville, IA). [1.5 $\mu \mathrm{g}]$ 505 plasmids were co-transfected with packaging plasmids pVSVg [0.5 $\mu \mathrm{g}$ ] and psPAX2 [1 $\mu \mathrm{g}]$ 506 Addgene plasmids \#8454, \#12260 in the presence of polyethylenimine (PEI) into HEK293T cells 507 for lentivirus production. $48 \mathrm{~h}$ post transfection virus was harvested and mixed with respective 508 cell line. Clones were selected for 7 days on [10 $\mu \mathrm{g} / \mathrm{ml}]$ Blasticidin and/or [1 $\mu \mathrm{g} / \mathrm{ml}]$ Puromycin. 509 KO cell lines were tested for CTxB binding by FACS and low binding population was sorted.

510 Cell lines carrying an ER version of Split-GFP were constructed according to Luong et al. (60) and 511 selected on Hygromycin [50 $\mathrm{gg} / \mathrm{ml}]$ for 7 days.

\section{Synthesis of peptide and fluorophore-labeled GM1 species}

513 Gangliosides of various fatty acid species were supplied by Prof. Sandro Sonnino (U. Milan,

514 Italy). Reporter peptide containing modified functional residues were custom synthesized by 515 New England Peptide (Gardner, MA) as all-D peptides with the following amino acid sequence: 516 propargylglycine-k(ع-biotinoyl)(ds)g(dy)g(dr)g(ds)g-(kaoa)-amine. Synthesis of peptide-lipid and 517 fluorophore labeled conjugates was accomplished with minor modifications according to 518 Garcia-Castillo et al., te Welscher et al. and Chinnapen et al. $(40,47,48)$. 
519 In a typical $2 \mathrm{ml}$ reaction, $2 \mathrm{mg}$ (approximately 1300 nmoles) of ganglioside was oxidized with 520 sodium periodate (13 mmoles) in oxidation buffer (100 mM sodium acetate $\mathrm{pH} 5.5,150 \mathrm{mM}$

$521 \mathrm{NaCl}$ ) for $30 \mathrm{~min}$ on ice. The reaction was quenched by addition of glycerol (5\% final) and 522 desalted using a Bond Elut SepPak C18 cartridge (Agilent, MA). Methanol was used to elute 523 oxidized GM1 species from the column and was removed by Speed Vac concentration (Savant).

524 The oxidized product was then reconstituted in $2 \mathrm{ml}$ PBS pH 6.9 in the presence of $10 \%$ DMF 525 and reacted with 2700 nmoles of aminooxy-containing peptide in the presence of $10 \mathrm{mM}$ 526 aniline (Dirksen and Dawson, 2008). The reaction was incubated for $20 \mathrm{~h}$ at room temperature 527 with mixing on a nutator. The precipitate was separated from the solution by centrifugation, 528 and then resuspended in $400 \mu \mathrm{l} 50 \%$ isopropanol/water after brief sonication. PBS pH 6.9 was 529 added $(200 \mu \mathrm{L})$ along with 4.8 mmoles of sodium cyanoborohydride and incubated for $3 \mathrm{~h}$ to 530 reduce the oxime bond. Lipid-peptide conjugates were purified by semi-preparative HPLC, and 531 confirmed by either MALDI-TOF (AB Voyager), or ESI LC-MS (Agilent, MA).

532 Peptides were labeled with Alexa fluorophore via copper mediated Click chemistry. 320 mM 533 peptide-lipid fusions containing an $\mathrm{N}$-terminal alkyne residue (propargylglycine) were reacted 534 with equimolar concentrations of AlexaFluor ${ }^{\mathrm{TM}}$-azide under the following conditions: $50 \mathrm{mM}$ 535 Tris-Cl, 5 mM copper (II) sulfate, 100 mM sodium ascorbate, 37 mM (Tris[(1-benzyl-1H-1,2,3536 triazol-4-yl)methyl]amine, TBTA in DMSO/t-butanol 1:4) $1 \mathrm{mM}$ (Tris(2-carboxyethyl) phosphine 537 hydrochloride, TCEP - Sigma) for $16 \mathrm{~h}$ at room temperature on a nutator. Products were 538 purified by HPLC and confirmed by mass spectrometry. Products were lyophilized and stored at $539-20^{\circ} \mathrm{C}$ or $-80^{\circ} \mathrm{C}$ for long term storage.

540 Working stock solutions were prepared in 33\% DMF/ddH20.

541 Alternatively, fatty acyl variant gangliosides were fluorophore labeled directly via the 542 oligosaccharide head group following the oxidation of the ganglioside. The oxidized 543 gangliosides were resuspended in phosphate buffered saline $\mathrm{pH} 7.4$ (PBS) and reacted with 1 544 mole AlexaFluor ${ }^{\mathrm{TM}}$-azide (Invitrogen, OR) for $16 \mathrm{~h}$. Ten moles sodium cyanoborohydride was 545 added to the reaction for 15 minutes and the mixture was desalted on Bond-Elute cartridges. 546 Ganglioside-fluorophore conjugates resulted in a different mobility band visible by TLC and 
547 were purified using preparative-TLC plates. Extraction from silica scrapings was performed, and

548 the conjugates further purified by reversed phase C18 cartridges.

\section{FACS based assay for lipid recycling, retrograde and lysosomal trafficking}

550 Cell lines were plated on 96 well plates and grown to $70 \%$ confluency (unless otherwise stated)

551 with or without treatment. Cells were washed and equilibrated with serum-free DMEM (no

552 phenol red) for $5 \mathrm{~min}$ at $37^{\circ} \mathrm{C}$. GM1 species were diluted to four different concentrations of the

553 respective peptide-labeled GM1 species in serum-free DMEM with equimolar ratio of dfBSA

554 (1:1 lipid:dfBSA). Lipids were loaded for $10 \mathrm{~min}$ at $37^{\circ} \mathrm{C}$ and washed of in serum-free DMEM (no

555 phenol red). To measure the amount of 'loaded' GM1, cells were trypsinized for 5 min at $37^{\circ} \mathrm{C}$,

556 then chilled to $4^{\circ} \mathrm{C}$ and stained with respective streptavidin-AlexaFluor ${ }^{\mathrm{TM}}(1: 2500,2 \mathrm{mg} / \mathrm{ml})$ in

557 PBS containing $5 \% \mathrm{BSA}$ for $15 \mathrm{~min} / 4^{\circ} \mathrm{C}$. Unbound streptavidin was washed off twice with PBS

558 containing 5\% FBS and cells were FACS analyzed immediately, using a FACS Canto II (BD,

559 Biosciences, NJ). Lipid+ gate was determined against streptavidin stained control cells, not

560 loaded with GM1. The percentage of positive cells and their median fluorescence were

561 recorded. To compare the recycling efficiency between the different GM1 variants, the

562 respective concentrations resulting in equal median fluorescence or equal \% of cells for the

563 'load' were chosen. To obtain 'chase' samples, the lipid was washed off and cells were

564 subsequently incubated for $3 \mathrm{~h}$ in DMEM containing 5\% FBS in the presence of Dextran-Fluor

$565(500 \mu \mathrm{g} / \mathrm{ml})$ and transferrin-AlexaFluor ${ }^{\mathrm{TM}}(1 \mu \mathrm{g} / \mathrm{ml})$. Cells were then washed and trypsinized and

566 stained with streptavidin-AlexaFluor ${ }^{\mathrm{TM}}$ as described above. To compare recycling rates between

567 different treatments, recycling rates (median fluorescence) for non-treated or WT cells were

568 normalized to 1 and fold change difference to treated samples calculated.

569 To determine retrograde trafficking of CTxB-SplitGFP, native (non-peptide labeled) GM1 species

570 were loaded as above into either Hela cells or an A431 CRISPR-Cas9 UGCG-KO cell line. 'Load'

571 amount of lipid was determined using 5nM fluorescently labeled CTxB instead of streptavidin as

572 described above. 'Chase' samples were incubated in the presence of $10 \mathrm{nM}$ CTxB-SplitGFP for

573 the indicated amount of time in DMEM containing 5\% FBS. For retrograde trafficking in CRISPR- 
574 Cas9 KO cell lines, cell surface GM1 amount was determined using 5nM fluorescently labeled

575 CTxB as described above and CTxB-SplitGFP values were normalized to cell surface GM1.

576 To measure lysosomal trafficking of GM1-CTxB complex, $\mathrm{pH}$ sensitive CTxB-pHrodo ${ }^{\text {TM }}$ was used.

577 Cells were chilled to $4^{\circ} \mathrm{C}$ and stained using $5 \mathrm{nM} \mathrm{CTxB-pHrodo}{ }^{\mathrm{TM}}$ in PBS containing 5\% BSA.

578 Unbound CTXB was washed off and cells were incubated for the indicated amount of time at

$57937^{\circ} \mathrm{C}$ in in DMEM containing 5\% FBS. Cell surface GM1 was determined using 5nM fluorescently

580 labeled CTxB as described above and CTxB-pHrodo ${ }^{\mathrm{TM}}$ values were normalized to cell surface

581 GM1.

582 Two biological replicates per assay were performed and a total of $N=10,000-20,000$ single cells 583 recorded per treatment for all FACS based assays.

584 Lysosomal trafficking of EGF receptor and LDL receptor

585 Cells were grown as described above. To determine the lysosomal fraction of different

586 membrane protein receptors, cells were loaded with their respective ligands at $(2-5 \mu \mathrm{g} / \mathrm{ml})$,

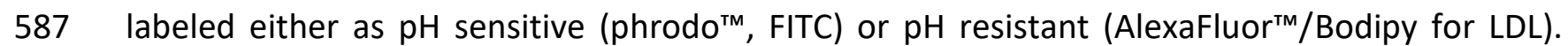

588 'Load' was determined by incubating pre-chilled cells for $10 \mathrm{~min}$ at $4^{\circ} \mathrm{C}$ with pH resistant ligand.

589 Cells were subsequent washed in PBS and detached using Cell Dissociation Buffer (Gibco).

590 Lysosomal fraction was determined by loading cells with $\mathrm{pH}$ sensitive ligand as above; after 591 washing off unbound ligand, cells were moved to $37^{\circ} \mathrm{C}$ for $2 \mathrm{~h}$ in the presence of fluorescent 592 dextran to normalize endocytosis rates. Cells were trypsinized and subsequently analyzed by 593 FACS.

594 Lipid loading, imaging and quantification for Airyscan or lattice lightsheet imaging

595 Cell lines were plated on either $35 \mathrm{~mm}$ MatTek petri dishes (Ashland, MA) containing glass 596 bottom for Airyscan confocal microscopy or on $3 \mathrm{~mm}$ cover slips for Lattice Lightsheet 597 microscopy and grown to 70 \% confluency with or without treatment. Fluorescent GM1 variants 598 were added as described for the FACS based assays for lipid recycling unless otherwise noted. 599 To determine subcellular location, lipids were allowed to traffic for the indicated amount of 
600 time at $37^{\circ} \mathrm{C}$ in the presence of fluorescent transferrin $(1 \mu \mathrm{g} / \mathrm{ml})$ and lysoTracker ${ }^{\mathrm{TM}}(2 \mathrm{nM})$ or 601 dextran $(1 \mathrm{mg} / \mathrm{ml})$ to demarcate the respective organelles. Sorting endosomes were defined as 602 vesicles containing lipid as well as transferrin and dextran. Sorting endosomal tubules were 603 defined by presence of transferrin emerging from triple positive vesicles.

604 For lactose and sucrose addition experiments A431 cells were incubated for $30 \mathrm{~min}$ in the 605 presence of $100 \mathrm{mM}$ lactose or sucrose prior/during and after GM1 loading.

606 Airyscan imaging was performed using Zeiss LSM 880 microscope with Airyscan detector and 607 Plan-Apochromat $63 x$ oil immersion objective with NA $=1.4$. Airyscan processing was 608 performed using the Zeiss ZenBlack software.

609 Quantification of GM1 in recycling endosomal tubules:

610 Region of interest (ROI) was determined, containing approximately 3 - 6 triple positive vesicles 611 and the ROI was thresholded using image quantification software ImageJ (Otsu). GM1 612 presence/absence (1/0) within transferrin positive tubules was recorded and \% for the ROI 613 calculated.

614 Tubule over vesicle fluorescence quantification:

615 For GM1 C14:0 and C22:1 $1^{\Delta 13}$ as well as for samples treated with cholesterol lowering molecules,

616 or cholesterol addition, fluorescence grey values for the vesicle and tubule were recorded, 617 background fluorescence was subtracted and a ratio was determined by dividing the mean grey 618 value obtained for the tubule by the mean grey value obtained for the vesicle (Fig. 1H). The 619 same analysis was performed for Fig. 2G and Suppl. Fig. 4D.

620 Quantification of endocytic rates:

621 Endocytic rates were assayed using CRISPR-Cas9 modified SVGA cell line containing an 622 endogenous, RAB5-EGFP variant (81). GM1 variants were loaded as described above, washed 623 off and immediately transferred to the microscope. Cells were imaged for approx. 5 min each. 
624 Vesicles were counted using ImageJ software and quantification was based on at least 5 625 biological replicates.

626 GM1 - Tfn co-localization in Rab5 enlarged early endosomes:

627 Using Fiji segmented line tool Rab5 early endosomes were traced using Rab5 fluorescence as 628 marker and mean grey fluorescence intensity profiles of GM1 variants and Tfn were exported 629 into GraphPad Prism. Pearson's correlation coefficient was calculated between different GM1 630 or Rab5 fluorescence profiles against Tfn fluorescence profile.

\section{Fluorescence recovery after bleaching (FRAP)}

632 Cell lines were plated on $35 \mathrm{~mm}$ MatTek petri dishes containing glass bottom $48 \mathrm{~h}$ prior to 633 experiments and treated as described above. GM1 lipids were incorporated into plasma 634 membranes as described above.

635 Cells were immediately transferred into preheated chamber for bleaching using the following 636 settings: 80 - 100 \% laser intensity, pixel dwell time of approx. $170 \mu \mathrm{sec}$, membrane 637 fluorescence was bleached to about $20-40 \%$ of the original fluorescence intensity. Three ROls 638 of similar size were chosen, one background ROI (ROI $\mathrm{bg}_{\mathrm{bg}}$ ) and two ROIs within the PM of a given 639 cells, with one of the latter being bleached $\left(\mathrm{ROI}_{\mathrm{b}}\right)$, while the other served as reference 640 photobleaching control $\left(\mathrm{ROI}_{\mathrm{nb}}\right)$. To establish the baseline, three - five frames were collected 641 before the bleaching event and to capture the recovery, at least fifteen frames were acquired 642 every 150 - 300 msec for a total time of approx. 10 sec post bleaching event.

643 FRAP curves were determined using the following formulas with $\mathrm{pb}=$ pre bleached fluorescence 644 (82):

645 (1) Bleached Fraction $(t)=1-\left[\frac{\left(R O I_{b}(t)-R O I_{b g}\right)}{\left(R_{n} I_{n b}(t)-R O I_{b g}\right)} * \frac{\left(p b R O I_{n b}-p b R O I_{b g}\right)}{\left(p_{b R O I_{b}}-R_{0 I} I_{b g}\right)}\right]$ 
$646 \quad(2)$

647 Fluorescence Recovery $=$

648 Bleached Fraction $\left(t_{\max }\right)-$ Bleached Fraction $\left(t_{\text {right after bleach }}\right)$

649

(3) Mobile Fraction $=\frac{\text { Fluorescence Recovery }}{\text { Bleached Fraction( } t_{\text {right after bleach })}}$

651 Cells were seeded two days prior to assay on 12 well plates to reach desired confluency. Native 652 or fluorescently labeled GM1 species were added as described above, and a phase marker 653 (FASTDiO $^{\mathrm{TM}}$ and FASTDiL ${ }^{\mathrm{TM}}, 0.5 \mathrm{mg} / \mathrm{ml}$ - 1. 1:1000 in PBS) was added for $10 \mathrm{~min}$. Phase marker 654 was washed off three times using PBS, then cells were equilibrated by washing twice in GPMV 655 buffer (50 mM Tris, $\mathrm{pH}=8,150 \mathrm{mM} \mathrm{NaCl}, 50 \mathrm{mM} \mathrm{CaCl} 2$ ). Vesiculation was achieved according 656 to (50) by using 2 mM DTT and $0.07 \%$ PFA in GPMV buffer and cells were incubated for 1-3 $\mathrm{h}$ at $65737^{\circ} \mathrm{C}$ (depending on the cell type). Supernatant containing GPMV vesicles were harvested and 658 sedimented $5 \mathrm{~min} / 500 \mathrm{~g}$. Vesicles were chilled to $10^{\circ} \mathrm{C}$ and imaged. $L_{d}$ phase was determined 659 by the phase marker and $L_{0}$ partitioning coefficient (ordered phase partitioning coefficient $=I_{10}$ ) 660 was obtained using the following formula: $K_{\text {raft }}=I_{L o} / I_{L d} . A K_{l o}>1$ indicates ordered phase 661 preference.

662 For membrane fluidity analysis, GPMVs were harvested from respective cells and/or treatments 663 as above and subsequently stained using [0.5 $\mu \mathrm{M}]$ laurdan for $3 \mathrm{~h}$ at RT. Dye was washed off 664 twice and vesicles were chilled to $10^{\circ} \mathrm{C}$ prior to imaging. Imaging was achieved by using spectral 665 imaging with confocal microscope Zeiss LSM 880 using Plan-Apochromat 63x oil immersion 666 objective (NA 1.4). C-laurdan was excited at $405 \mathrm{~nm}$ and the emission was collected with a 667 multichannel spectral detector (PMT) in the range $430-450 \mathrm{~nm}$ and $480-500 \mathrm{~nm}$ 668 simultaneously. The polarity index, generalized polarization (GP) was calculated according to 669 Steinkuehler et al. (68) using the following formula: GP $=\left(I_{440}-I_{490}\right) /\left(I_{440}+I_{490}\right)$ where $I_{440}$ and $670 \mathrm{I}_{490}$ are the fluorescence intensities at $440 \mathrm{~nm}$ and $490 \mathrm{~nm}$, respectively. 
672 Lipid extraction and Lipidomic profiling of A431 WT and CRISPR-Cas9 KO cell lines

673500,000 cells per biological/technical replicate were washed twice with PBS and then $0.25 \times$ PBS

674 to remove media components and transferred into glass vials. Cells were then pelleted to

675 remove remaining supernatant and flash frozen in liquid nitrogen and pellet was subsequently

676 lyophilized. Lipid extraction and lipidomic profiling was performed according to Liaw et al. and

677 Kumar et al. $(83,84)$. In brief, the lyophilized pellet was homogenized in $1 \mathrm{ml} d d \mathrm{H}_{2} \mathrm{O}$,

678 subsequently $2.2 \mathrm{ml}$ methanol and $0.9 \mathrm{ml}$ dichlormethane was added. Suspension was

679 incubated o/N at -20C, before $20 \mu \mathrm{l}$ Avanti Splash mix was added, mixture was vortexed and

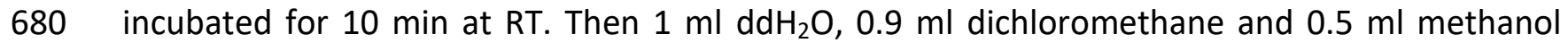

681 were added. Solution was centrifuged at $1200 \mathrm{rpm}$ for $10 \mathrm{~min}$ and lower phase collected into

682 fresh glass tube. $2 \mathrm{ml}$ dichloromethane were added to the aqueous phase to extract any

683 remaining lipids, mixture was centrifuged as above and the organic phase was pooled with the

684 first one.

685 Solvent was evaporated in Genevac.

686 Unbiased MS/MSALL shotgun lipidomic profiling was performed on Sciex 5600 TripleTOF mass 687 spectrometer.

688 Software and Statistical analysis

689 Imaging data was analyzed using either Zeiss ZENblack, Fiji or Aiviva 9 (Drvision Technologies,

690 Bellevue, WA) software. Lattice lightsheet data obtained at the Janelia Farms Advanced Imaging 691 center (AIC), deskewed and decovoluted using customized Matlab ${ }^{\text {TM }}$ software.

692 Schematic Model in Fig. 1A was drawn using BioRender.

693 Mean grey fluorescent values of microscopy experiments or mean fluorescence data obtained

694 from FACS experiments was transferred to GraphPad Prism software (San Diego, CA) for 695 graphing and statistical analysis. Statistically significant difference between treatments was 696 tested by One-way ANOVA with Tukey's multiple comparison; alternatively unpaired t-test was 697 applied when indicated with 
n.s. $\mathrm{p}>0.05, * \mathrm{p}<0.05, * * \mathrm{p}<0.01 * * * \mathrm{p}<0.001$ and $* * * * \mathrm{p}<0.0001$

Table 1

701 Guide sequences were used from AVANA sgGuide library (85).

\begin{tabular}{|c|c|}
\hline Gene & sgGuide sequence \\
\hline SPTLC-2 & ACAACTATCTTGGATTTGCA \\
\hline UGCG & ACAACTATCTTGGATTTGCA \\
\hline \multirow[t]{4}{*}{ CerS1 } & AGCGCTCTCGGGCATCTTGG \\
\hline & GCTTCCCTGGAGCAGGTAGG \\
\hline & GCAGCCGCCTACCTGCTCCA \\
\hline & TACGCGCAGCTAGTGCAGC \\
\hline \multirow[t]{4}{*}{ CerS2 } & GCTGCCTGTGAACTTGACCT \\
\hline & TTCTTCATGTCATAGAACCA \\
\hline & GGTCAGGTAGAAATGTTCCA \\
\hline & GTAAAATGTGAATCTCCAGC \\
\hline \multirow[t]{4}{*}{ CerS5 } & GTATCCTGATAAGAAAAGGC \\
\hline & TGATGAAACCAAGACCCAAG \\
\hline & ATGCCAATACAGAGTGCACA \\
\hline & ATAAAATGTGAATCTCCACC \\
\hline
\end{tabular}




\section{References}

704

705

706

707

708

709

710

711

712

713

714

715

716

717

718

719

720

721

722

723

724

725

726

727

728

729

730

731

732

733

734

735

736

737

738

739

740

741

742

743

744

745

746

747
1. M. Koval, R. E. Pagano, Intracellular transport and metabolism of sphingomyelin. Biochimica et biophysica acta 1082, 113 (Mar 12, 1991).

2. G. van Meer, K. Simons, Viruses budding from either the apical or the basolateral plasma membrane domain of MDCK cells have unique phospholipid compositions. The EMBO journal $\mathbf{1}$, 847 (1982/07/01, 1982).

3. B. Ramstedt, J. P. Slotte, Membrane properties of sphingomyelins. FEBS Lett 531, 33 (Oct 30, 2002).

4. S. Chiantia, E. London, in Sphingolipids: Basic Science and Drug Development, E. Gulbins, I. Petrache, Eds. (Springer Vienna, Vienna, 2013), pp. 33-55.

5. D. A. Brown, E. London, Structure and origin of ordered lipid domains in biological membranes. $J$ Membr Biol 164, 103 (Jul 15, 1998).

6. E. London, How principles of domain formation in model membranes may explain ambiguities concerning lipid raft formation in cells. Bba-Mol Cell Res 1746, 203 (Dec 30, 2005).

7. D. Goswami et al., Nanoclusters of GPI-anchored proteins are formed by cortical actin-driven activity. Cell 135, 1085 (Dec 12, 2008).

8. K. Gowrishankar et al., Active remodeling of cortical actin regulates spatiotemporal organization of cell surface molecules. Cell 149, 1353 (Jun 8, 2012).

9. A. Kusumi et al., Membrane mechanisms for signal transduction: the coupling of the meso-scale raft domains to membrane-skeleton-induced compartments and dynamic protein complexes. Seminars in cell \& developmental biology 23, 126 (Apr, 2012).

10. C. A. Day, A. K. Kenworthy, Mechanisms underlying the confined diffusion of cholera toxin Bsubunit in intact cell membranes. PLoS One 7, e34923 (2012).

11. A. Fujita, J. Cheng, T. Fujimoto, Segregation of GM1 and GM3 clusters in the cell membrane depends on the intact actin cytoskeleton. Biochimica et biophysica acta 1791, 388 (May, 2009).

12. L. Johannes, R. G. Parton, P. Bassereau, S. Mayor, Building endocytic pits without clathrin.

13. L. J. Pike, Lipid rafts: heterogeneity on the high seas. The Biochemical journal 378, 281 (Mar 1, 2004).

14. L. Johannes, R. G. Parton, P. Bassereau, S. Mayor, Building endocytic pits without clathrin. Nat Rev Mol Cell Biol 16, 311 (May, 2015).

15. D. Lingwood, K. Simons, Lipid rafts as a membrane-organizing principle. Science 327,46 (Jan 1, 2010).

16. K. Jacobson, P. Liu, B. C. Lagerholm, The Lateral Organization and Mobility of Plasma Membrane Components. Cell 177, 806 (May 2, 2019).

17. K. Raghunathan, A. K. Kenworthy, Dynamic pattern generation in cell membranes: Current insights into membrane organization. Biochim Biophys Acta Biomembr 1860, 2018 (Oct, 2018 ).

18. E. Sezgin, I. Levental, S. Mayor, C. Eggeling, The mystery of membrane organization: composition, regulation and roles of lipid rafts. Nat Rev Mol Cell Biol 18, 361 (Jun, 2017).

19. K. Simons, J. L. Sampaio, Membrane organization and lipid rafts. Cold Spring Harb Perspect Biol 3, a004697 (Oct 1, 2011).

20. K. Simons, G. van Meer, Lipid sorting in epithelial cells. Biochemistry 27, 6197 (1988).

21. P. Varshney, V. Yadav, N. Saini, Lipid rafts in immune signalling: current progress and future perspective. Immunology 149, 13 (Sep, 2016).

22. Y. Zhou, J. F. Hancock, Ras nanoclusters: Versatile lipid-based signaling platforms. Biochimica et biophysica acta 1853, 841 (Apr, 2015). 
23. M. F. Garcia-Parajo, A. Cambi, J. A. Torreno-Pina, N. Thompson, K. Jacobson, Nanoclustering as a dominant feature of plasma membrane organization. Journal of cell science 127, 4995 (2014).

24. J. Lippincott-Schwartz, R. D. Phair, Lipids and cholesterol as regulators of traffic in the endomembrane system. Annu Rev Biophys 39, 559 (2010).

752

753

754

755

25. G. H. Patterson et al., Transport through the Golgi apparatus by rapid partitioning within a twophase membrane system. Cell 133, 1055 (Jun 13, 2008).

26. Y. Deng, F. E. Rivera-Molina, D. K. Toomre, C. G. Burd, Sphingomyelin is sorted at the trans Golgi network into a distinct class of secretory vesicle. Proc Natl Acad Sci U S A 113, 6677 (Jun 14, 2016).

27. M. B. Stone, S. A. Shelby, M. F. Nunez, K. Wisser, S. L. Veatch, Protein sorting by lipid phase-like domains supports emergent signaling function in B lymphocyte plasma membranes. Elife 6 , e19891 (Feb 1, 2017).

28. W. I. Lencer, B. Tsai, The intracellular voyage of cholera toxin: going retro. Trends in biochemical sciences 28, 639 (Dec, 2003).

29. P. Cuatrecasas, Gangliosides and membrane receptors for cholera toxin. Biochemistry 12, 3558 (Aug 28, 1973).

30. M. A. Campanero-Rhodes et al., N-glycolyl GM1 ganglioside as a receptor for simian virus 40. J Virol 81, 12846 (Dec, 2007).

31. K. Sandvig, Shiga toxins. Toxicon 39, 1629 (Nov, 2001).

32. M. Lorizate et al., Comparative lipidomics analysis of HIV-1 particles and their producer cell membrane in different cell lines. Cell Microbiol 15, 292 (Feb, 2013).

33. W. Romer et al., Shiga toxin induces tubular membrane invaginations for its uptake into cells. Nature 450, 670 (Nov 29, 2007).

34. R. Lakshminarayan et al., Galectin-3 drives glycosphingolipid-dependent biogenesis of clathrinindependent carriers. Nat Cell Biol 16, 595 (Jun, 2014).

35. Y. A. Hannun, L. M. Obeid, Sphingolipids and their metabolism in physiology and disease. Nat Rev Mol Cell Biol 19, 175 (Mar, 2018).

36. A. H. Merrill, Jr., Sphingolipid and glycosphingolipid metabolic pathways in the era of sphingolipidomics. Chem Rev 111, 6387 (Oct 12, 2011).

37. W. W. Young, M. S. Lutz, W. A. Blackburn, Endogenous glycosphingolipids move to the cell surface at a rate consistent with bulk flow estimates. Journal of Biological Chemistry 267, 12011 (1992/06/15/, 1992).

38. G. van Meer, D. R. Voelker, G. W. Feigenson, Membrane lipids: where they are and how they behave. Nat Rev Mol Cell Biol 9, 112 (Feb, 2008).

39. D. E. Saslowsky et al., Ganglioside GM1-mediated transcytosis of cholera toxin bypasses the retrograde pathway and depends on the structure of the ceramide domain. J Biol Chem 288, 25804 (Sep 6, 2013).

40. D. J. Chinnapen et al., Lipid sorting by ceramide structure from plasma membrane to ER for the cholera toxin receptor ganglioside GM1. Developmental cell 23, 573 (Sep 11, 2012).

41. S. Rodriguez-Gallardo et al., Ceramide chain length-dependent protein sorting into selective endoplasmic reticulum exit sites. Sci Adv 6, (Dec, 2020).

42. C. M. Paton, J. M. Ntambi, Biochemical and physiological function of stearoyl-CoA desaturase. American journal of physiology. Endocrinology and metabolism 297, E28 (Jul, 2009).

43. B. Ramstedt, J. P. Slotte, Interaction of cholesterol with sphingomyelins and acyl-chain-matched phosphatidylcholines: a comparative study of the effect of the chain length. Biophys J 76, 908 (Feb, 1999).

44. A. Kihara, Very long-chain fatty acids: elongation, physiology and related disorders. Journal of biochemistry 152, 387 (Nov, 2012). 
796

797

798

799

800

801

802

803

804

805

806

807

808

809

810

811

812

813

814

815

816

817

818

819

820

821

822

823

824

825

826

827

828

829

830

831

832

833

834

835

836

837

838

839

840

841

842

843

45. S. Kavaliauskiene et al., Cell density-induced changes in lipid composition and intracellular trafficking. Cellular and molecular life sciences : CMLS 71, 1097 (Mar, 2014).

46. R. L. Shaner et al., Quantitative analysis of sphingolipids for lipidomics using triple quadrupole and quadrupole linear ion trap mass spectrometers. J Lipid Res 50, 1692 (Aug, 2009).

47. Y. M. te Welscher, D. J. Chinnapen, L. Kaoutzani, R. J. Mrsny, W. I. Lencer, Unsaturated glycoceramides as molecular carriers for mucosal drug delivery of GLP-1. J Control Release 175, 72 (Feb 10, 2014).

48. M. D. Garcia-Castillo et al., Mucosal absorption of therapeutic peptides by harnessing the endogenous sorting of glycosphingolipids. Elife 7, e34469 (May 31, 2018).

49. S. L. Veatch, S. L. Keller, Separation of liquid phases in giant vesicles of ternary mixtures of phospholipids and cholesterol. Biophys J 85, 3074 (Nov, 2003).

50. E. Sezgin et al., Elucidating membrane structure and protein behavior using giant plasma membrane vesicles. Nat Protoc 7, 1042 (May 3, 2012).

51. F. R. Maxfield, T. E. McGraw, Endocytic recycling. Nat Rev Mol Cell Biol 5, 121 (Feb, 2004).

52. S. Mukherjee, R. N. Ghosh, F. R. Maxfield, Endocytosis. Physiol Rev 77, 759 (Jul, 1997).

53. S. B. Kellie, B. Patel, J. Pierce, D. Critchley, Capping of cholera toxin - Ganglioside GM1 complexes on mouse lymphocytes is accompanied by co-capping of alpha-actinin. J. Cell Biol. 97, 447 (1983).

54. A. M. Kabbani, K. Raghunathan, W. I. Lencer, A. K. Kenworthy, C. V. Kelly, Structured clustering of the glycosphingolipid GM1 is required for membrane curvature induced by cholera toxin. Proceedings Of The National Academy Of Sciences Of The United States Of America. 117, 14978 (Jun 30, 2020).

55. B. Sorre et al., Curvature-driven lipid sorting needs proximity to a demixing point and is aided by proteins. Proc Natl Acad Sci U S A 106, 5622 (Apr 7, 2009).

56. B. N. Stillman et al., Galectin-3 and galectin-1 bind distinct cell surface glycoprotein receptors to induce T cell death. Journal of immunology 176, 778 (Jan 15, 2006).

57. S. Mayor, J. F. Presley, F. R. Maxfield, Sorting of membrane components from endosomes and subsequent recycling to the cell surface occurs by a bulk flow process. J Cell Biol 121, 1257 (Jun, 1993).

58. A. A. Wolf et al., Attenuated Endocytosis and Toxicity of a Mutant Cholera Toxin with Decreased Ability to Cluster Gm1. Infect Immun 76, 1476 (Jan 22, 2008).

59. K. Raghunathan et al., Glycolipid Crosslinking Is Required for Cholera Toxin to Partition Into and Stabilize Ordered Domains. Biophys J, (Nov 30, 2016).

60. P. Luong et al., A quantitative single-cell assay for retrograde membrane traffic enables rapid detection of defects in cellular organization. Molecular biology of the cell 31, 511 (2020).

61. J. W. Park, W. J. Park, A. H. Futerman, Ceramide synthases as potential targets for therapeutic intervention in human diseases. Biochimica et biophysica acta 1841, 671 (May, 2014).

62. K. M. Spillane et al., High-speed single-particle tracking of GM1 in model membranes reveals anomalous diffusion due to interleaflet coupling and molecular pinning. Nano Lett 14, 5390 (Sep 10, 2014).

63. J. S. Goodwin, K. R. Drake, C. L. Remmert, A. K. Kenworthy, Ras diffusion is sensitive to plasma membrane viscosity. Biophys J 89, 1398 (Aug, 2005).

64. A. Roux et al., Role of curvature and phase transition in lipid sorting and fission of membrane tubules. The EMBO journal 24, 1537 (Apr 20, 2005).

65. T. Baumgart, S. T. Hess, W. W. Webb, Imaging coexisting fluid domains in biomembrane models coupling curvature and line tension. Nature 425, 821 (Oct 23, 2003).

66. S. Mukherjee, T. T. Soe, F. R. Maxfield, Endocytic sorting of lipid analogues differing solely in the chemistry of their hydrophobic tails. J Cell Biol 144, 1271 (Mar 22, 1999). 
844

67. P. Noutsi, E. Gratton, S. Chaieb, Assessment of Membrane Fluidity Fluctuations during Cellular Development Reveals Time and Cell Type Specificity. PLoS One 11, e0158313 (2016).

68. J. Steinkuhler, E. Sezgin, I. Urbancic, C. Eggeling, R. Dimova, Mechanical properties of plasma membrane vesicles correlate with lipid order, viscosity and cell density. Commun Biol 2, 337 (2019/09/13, 2019).

69. M. Hao, S. Mukherjee, Y. Sun, F. R. Maxfield, Effects of cholesterol depletion and increased lipid unsaturation on the properties of endocytic membranes. The Journal of biological chemistry 279, 14171 (Apr 2, 2004).

70. A. Kihara, Very long-chain fatty acids: elongation, physiology and related disorders.

71. M. L. Fanani, B. Maggio, The many faces (and phases) of ceramide and sphingomyelin I - single lipids. Biophys Rev 9, 589 (Oct, 2017).

72. M. L. Fanani, B. Maggio, The many faces (and phases) of ceramide and sphingomyelin II - binary mixtures. Biophys Rev 9, 601 (Oct, 2017).

73. G. W. Stockton, I. C. Smith, A deuterium nuclear magnetic resonance study of the condensing effect of cholesterol on egg phosphatidylcholine bilayer membranes. I. Perdeuterated fatty acid probes. Chem Phys Lipids 17, 251 (Oct, 1976).

74. S. Jaikishan, A. Bjorkbom, J. P. Slotte, Sphingomyelin analogs with branched $\mathrm{N}$-acyl chains: the position of branching dramatically affects acyl chain order and sterol interactions in bilayer membranes. Biochimica et biophysica acta 1798, 1987 (Oct, 2010).

75. K. Simons, W. L. Vaz, Model systems, lipid rafts, and cell membranes. Annu Rev Biophys Biomol Struct 33, 269 (2004/06/09, 2004).

76. R. Raghupathy et al., Transbilayer lipid interactions mediate nanoclustering of lipid-anchored proteins. Cell 161, 581 (Apr 23, 2015).

77. T. Fujimoto, I. Parmryd, Interleaflet Coupling, Pinning, and Leaflet Asymmetry-Major Players in Plasma Membrane Nanodomain Formation. Frontiers in Cell and Developmental Biology 4, 155 (2017).

78. T. Rog et al., Interdigitation of long-chain sphingomyelin induces coupling of membrane leaflets in a cholesterol dependent manner. Biochimica et biophysica acta 1858, 281 (Feb, 2016).

79. T. Skotland, K. Sandvig, The role of PS 18:0/18:1 in membrane function. Nat Commun 10, 2752 (Jun 21, 2019).

80. T. K. M. Nyholm et al., Impact of Acyl Chain Mismatch on the Formation and Properties of Sphingomyelin-Cholesterol Domains. Biophys J 117, 1577 (Nov 5, 2019).

81. Y. Y. Chou et al., Identification and Characterization of a Novel Broad-Spectrum Virus Entry Inhibitor. J Virol 90, 4494 (May, 2016).

82. M. Nissim-Rafinia, E. Meshorer, Photobleaching assays (FRAP \& FLIP) to measure chromatin protein dynamics in living embryonic stem cells. Journal of visualized experiments : JoVE, e2696 (Jun 29, 2011).

83. L. Liaw et al., Lipid Profiling of In Vitro Cell Models of Adipogenic Differentiation: Relationships With Mouse Adipose Tissues. J Cell Biochem 117, 2182 (Sep, 2016).

84. N. Gajenthra Kumar et al., Untargeted lipidomic analysis to broadly characterize the effects of pathogenic and non-pathogenic staphylococci on mammalian lipids. PLoS One 13, e0206606 (2018).

85. J. G. Doench et al., Optimized sgRNA design to maximize activity and minimize off-target effects of CRISPR-Cas9. Nat Biotechnol 34, 184 (Feb, 2016). 


\section{Acknowledgements}

890 We thank the Janelia Farms Advanced Imaging Center for providing access and support to their

891 Lattice Lightsheet microscope. Imaging data used in this publication was produced in

892 collaboration with the Advanced Imaging Center, a facility jointly supported by the Gordon and

893 Betty Moore Foundation and HHMI at HHMI's Janelia Research Campus. We further thank the

894 Harvard Center for Biological Imaging, Boston Children's Hospital Cell Function and Imaging

895 Core and HDDC Imaging Core for infrastructure and support.

896 We thank Prof. Dr. Kirchhausen (Boston Children's Hospital, Harvard Medical School, USA) for

897 providing the SVGA-Rab5-GFP cell line, as well as Prof. Dr. Waldor and Dr. Alline Pacheco

898 (Brigham and Woman's Hospital, Harvard Medical School, USA) for providing one CRISPR-Cas9

899 UGCG and SPTLC-2 KO plasmids and HT29 Cas9 positive cell line.

900 We further thank Dr. Daniel Chinnapen, Dr. Richard Duclos, Dr. Phi Luong and Dr. Jamie 901 LeBarron for helpful discussions during the preparation of this manuscript and Profs. 902 Thiagarajah (Boston Children's Hospital, Harvard Medical School, USA), Johannes (Institut Curie,

903 France) and Kenworthy (University of Virginia, School of Medicine, USA) for critically reading 904 the manuscript.

905 This work was supported by grants R37 DK048106 and RO1 DK104868 to WIL, and P30 906 DK034854 to the Harvard Digestive Disease Center

\section{Author contributions}

909 S.S.S and W.I.L. conceive study; S.S.S, R.T., W.I.L. designed experiments and resources; S.S.S,

910 R.T. and K.K. performed experiments; S.S.S, R.T., and M.A. analyzed data. S.S.S. and W.I.L., 911 wrote manuscript. 


\section{Figures}

A.

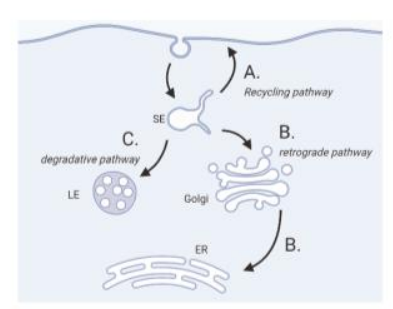

C.
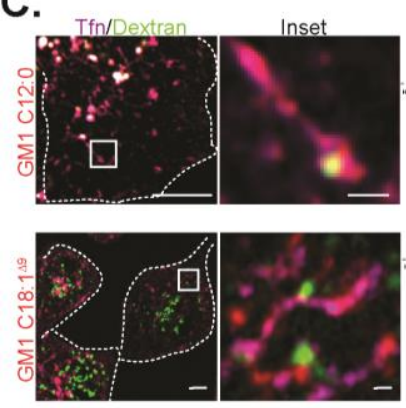

E.

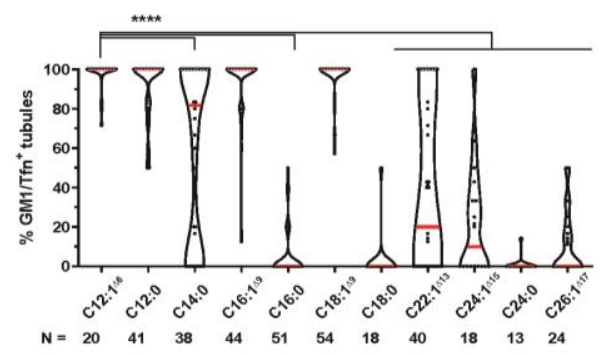

$\mathrm{H}$.

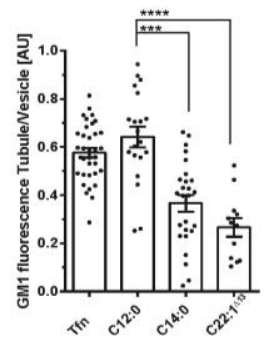

B.

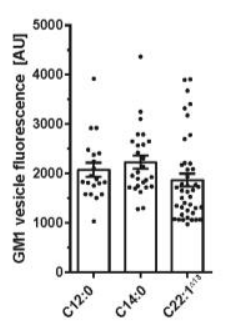

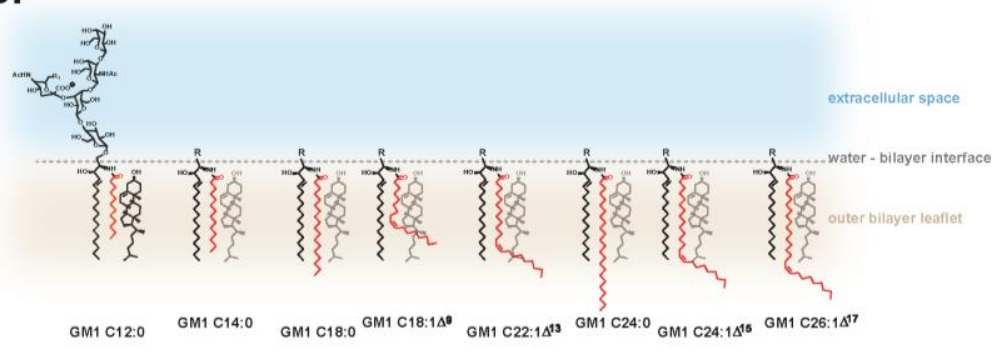

D.
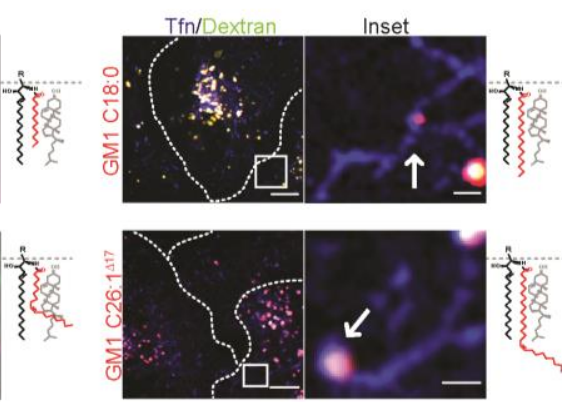

F.

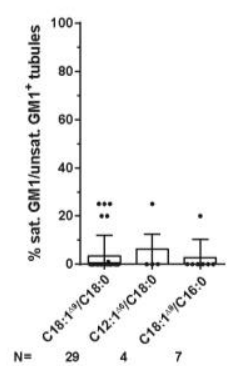

G.

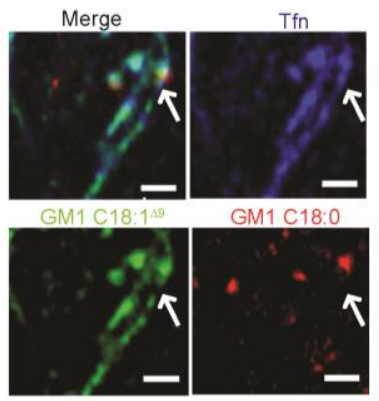

G. Merge Tfn/Dextran GM1 only

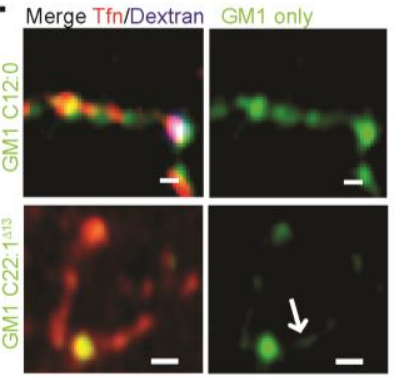

I.

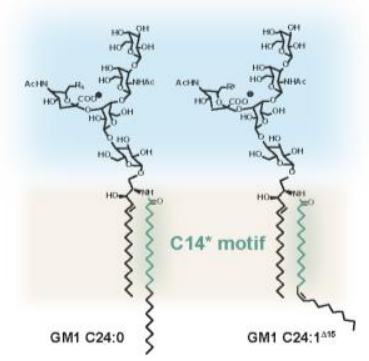

915 Fig. 1. Ceramide structure-specific trafficking within the sorting endosome. (A) Schematic of

916 endocytic pathways emerging from the sorting endosome (SE). Both the recycling and

917 retrograde pathways (marked $\mathrm{A}$ and $\mathrm{B}$ ) require sorting into narrow endosomal tubules. Cargos

918 destined for degradation are retained within the sorting endosomal body for maturation to late 
919 endosomes (LE) (marked C). (B) Library of GM1 species synthesized - acyl chains marked in red 920 and arbitrarily aligned against cholesterol to mark changes in structure. GM1 C12:1 ${ }^{\Delta 6}, \mathrm{C} 16: 0$ and

$921 \mathrm{GM} 1 \mathrm{C} 16: 1^{\Delta 9}$, species not shown. (C) Airyscan live cell imaging of recycling endosomal vesicles 922 in A431 epithelial cells. Scale bars $=5$ and $0.5 \mu \mathrm{m}$ respectively. (D) Same as panel $\mathrm{C}$ for cells 923 treated with GM1 C18:1 ${ }^{\Delta 9}$ (green) and GM1 C18:0 (red). Scale bar $=1 \mu \mathrm{m}$. (E) Quantification of 924 GM1 presence in Tfn marked recycling endosomal tubules. Mean \pm SEM, $* * * * p=<0.0001$ by 925 one-way ANOVA and Tukey's multiple comparison using GM1 C12:0 as comparison. (F) Co926 localization of GM1 C18:0 or C16:0 with GM1 C18:1 $1^{\Delta 9}$ or C12:1 ${ }^{\Delta 6}$ species in recycling Tfn-positive 927 endosomal tubules. Quantification and statistics as in panel E. (G) Recycling tubules analyzed as 928 in Panel C using GM1 C22:1 ${ }^{\Delta 13}$ and GM1 C12:0 species (green), Tfn (red). Scale bar $=0.5 \mu \mathrm{m}$. (H) 929 Left panel: Quantification of mean grey values of GM1 fluorescence in Tfn tubule normalized to $930 \mathrm{GM} 1$ fluorescence in body of recycling endosomes for GM1 C12:0, C14:0 and C22:1 ${ }^{\Delta 13}$. Right 931 panel: Mean grey value of GM1 fluorescence in body of recycling endosome for the same GM1 932 species. Mean \pm SEM. ${ }^{* * * *} \mathrm{p}=<0.0001$ against GM1 C12:0 by One-way ANOVA and Tukey's 933 multiple comparison. (I) GM1 C24:0 and GM1 C24:1 ${ }^{\Delta 15}, \mathrm{C} 14^{*}$ motif in turquoise. 
A.

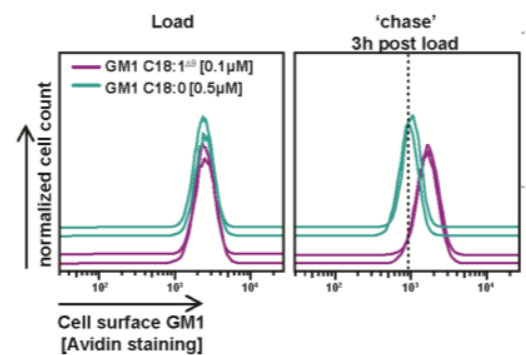

B.

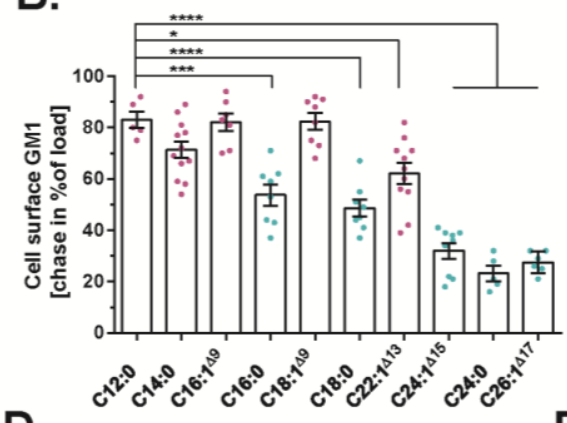

D.

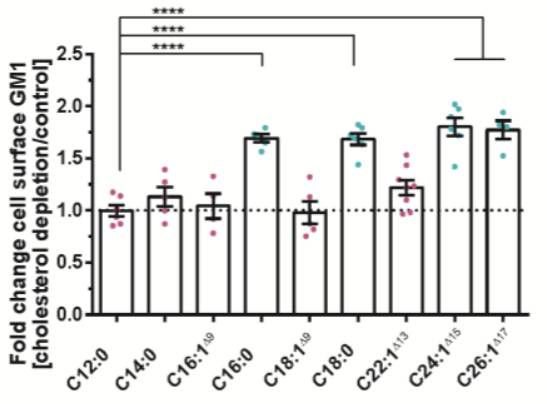

F.

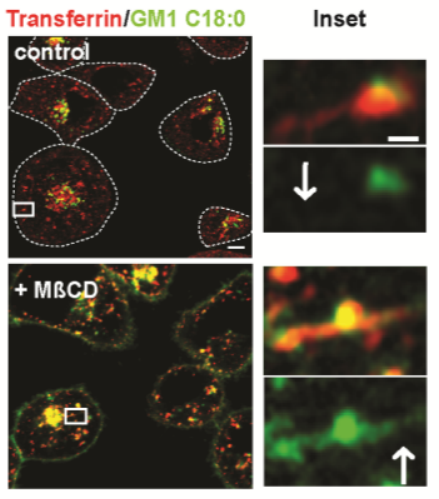

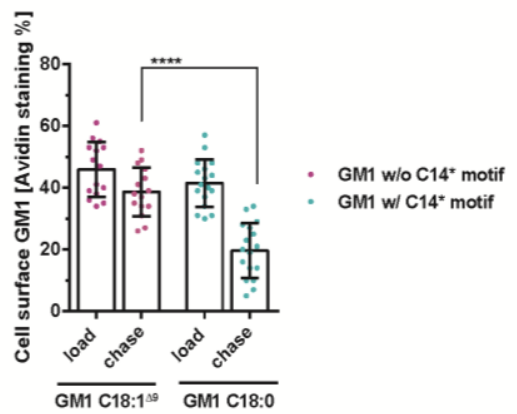

C.

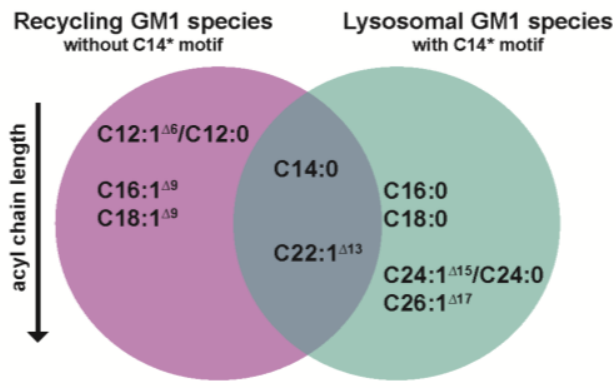

E.

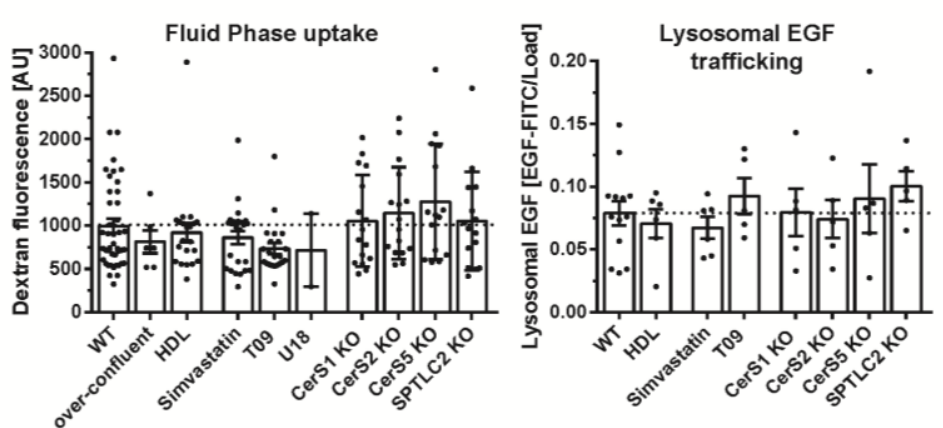

G.

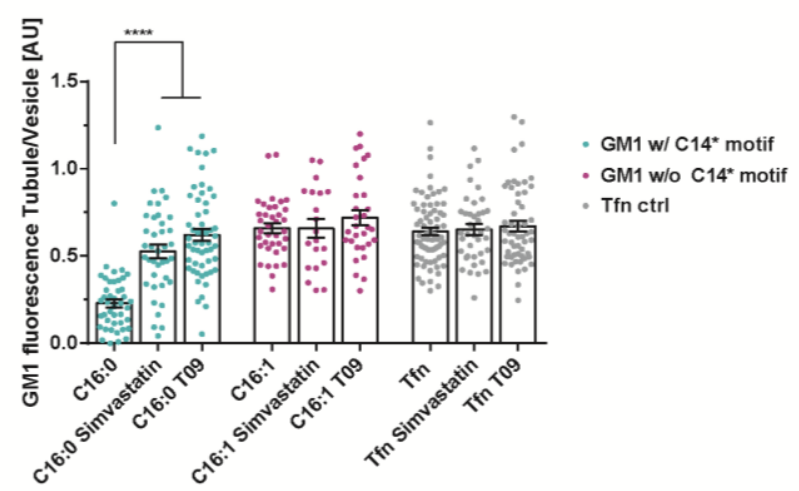


936 (A) PM concentrations of GM1 C18:0 (turquoise, with C14* motif) and GM1 C18:1 ${ }^{\Delta 9}$ (magenta, 937 without C14* motif) as assessed by FACS using AlexaFluor ${ }^{\mathrm{TM}}$-labeled streptavidin before and 938 after $3 \mathrm{~h}$ chase. Mean \pm SEM (right panel), each dot equals 2 biological replicates measuring 93920,000 cells each. $* * * * p=<0.0001$ by unpaired t-test. (B) Plasma membrane recycling as in 940 panel A for the GM1 structural library (GM1 species with C14* motif in turquoise, GM1 species 941 without C14* motif in magenta). Mean \pm SEM. One-way ANOVA and Tukey's multiple 942 comparison. (C) Venn diagram for ceramide acyl chain structure dependent GM1 sorting in the 943 endosomal system. Recycling GM1 species, without C14* motif are in magenta and GM1 944 species entering the lysosomal pathway, containing the C14* motif are in turquoise. (D) Change 945 in recycling rates under mildly cholesterol depleting conditions as indicated. $* * * * p=<0.0001$ 946 by One-way ANOVA and Tukey's multiple comparison. (E) Fluid phase uptake (left) and 947 lysosomal trafficking (right) measured by FACS in A431 cells depleted or not of cholesterol as 948 indicated. No differences were observed as measured by One-way ANOVA and Tukey's multiple 949 comparison. (F) Images of recycling tubules in A431 cells treated or not with M $\beta C D$. Scale bars = 9505 and $0.5 \mu \mathrm{m}$ respectively. (G) Entry of GM1 C16:0 or $\mathrm{C} 16: 1^{\Delta 9}$ into recycling tubules in 951 cholesterol depleted A431 cells as indicated. Mean grey values of fluorescent GM1 in both 952 endosomal vesicle as well as tubule was recorded and the ratio of GM1 fluorescence in tubule 953 over vesicle was calculated for each condition. Each dot represents one tubule vs vesicle ratio.

954 Mean \pm SEM. ${ }^{* * * *} \mathrm{p}=<0.0001$ by One-way ANOVA and Tukey's multiple comparison. 
A.

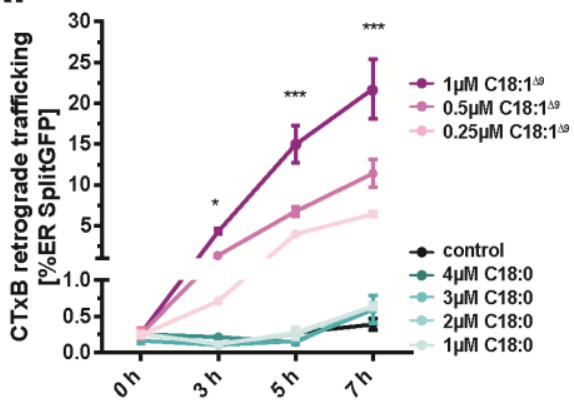

C.

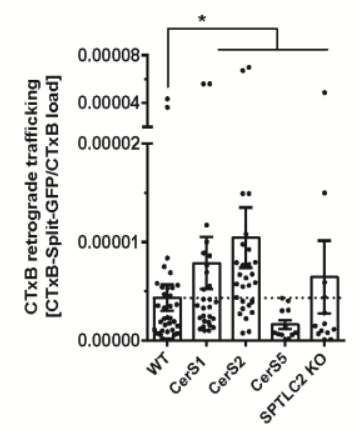

D.

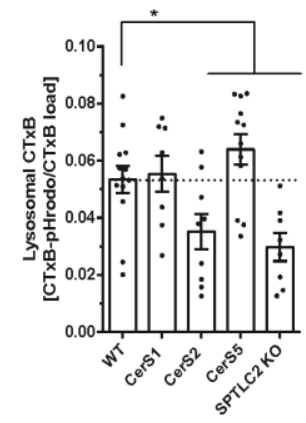

B.

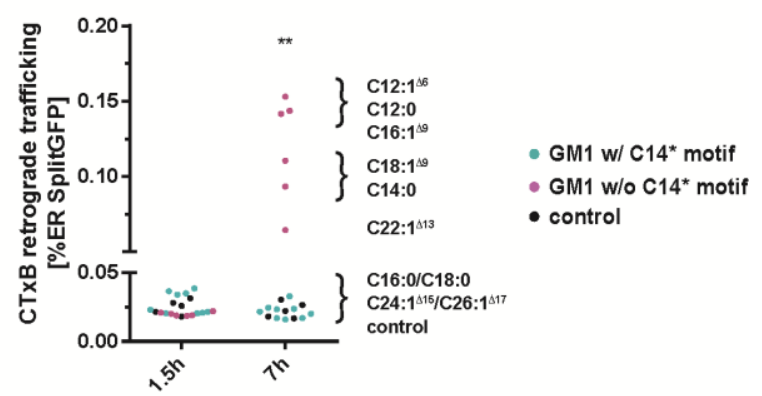

E.

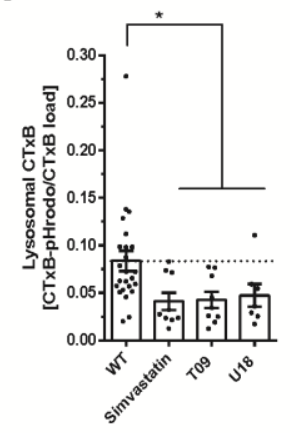

F.

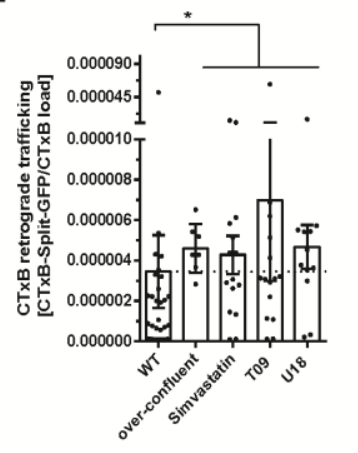

Fig. 3. Ceramide-dependent sorting into the retrograde pathway. (A) Time course of retrograde GM1 trafficking to the ER by a SplitGFP based FACS assay Graph dots represent the mean \pm SD of 2 biological replicates measuring 20,000 cells each. ${ }^{* * * *} p=<0.0001$ by One-way ANOVA and Tukey's multiple comparison. (B) SplitGFP assay as in (A) loaded with different GM1 species. Graph dots represent the mean \pm SEM of 2 biological replicates measuring 20,000 cells each. (C) A431 CRISPR-Cas9 KO cell lines for different ceramide synthases stably expressing ER-

963 One-way ANOVA and Tukey's multiple comparison. (D) FACS assay measuring lysosomal 964 transport of GM1-CTXB-pHrodo ${ }^{T M}$ in A431 CRISPR-Cas9 KO cell lines for different ceramide. Dot 965 represents mean of 3 biological replicates of 20,000 cells each. Mean \pm SEM. ${ }^{* * * *} p=<0.0001$ 966 by One-way ANOVA and Tukey's multiple comparison. (E) Lysosomal transport of GM1-CTxB967 pHrodo $^{T M}$ in A431 WT cells mildly depleted of cholesterol as indicated. Dot represents mean of 9683 biological replicates measuring 20,000 cells each. Mean \pm SEM. $* * * * p=<0.0001$ by One-way 969 ANOVA and Tukey's multiple comparison. (F) A431 WT cells were mildly depleted of cholesterol 970 as indicated and retrograde transport was measured. Dot represents mean of 3 biological 
bioRxiv preprint doi: https://doi.org/10.1101/2021.04.26.440603; this version posted April 26, 2021. The copyright holder for this preprint (which was not certified by peer review) is the author/funder, who has granted bioRxiv a license to display the preprint in perpetuity. It is made available under aCC-BY-NC-ND 4.0 International license.

971 replicates measuring 20,000 cells each. Mean \pm SEM. $* * * * p=<0.0001$ by One-way ANOVA and

972 Tukey's multiple comparison.

A.
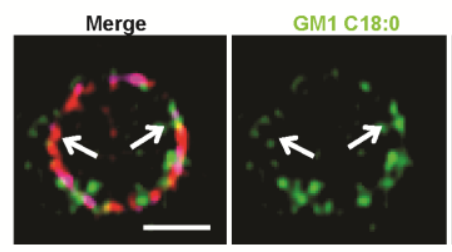

C.

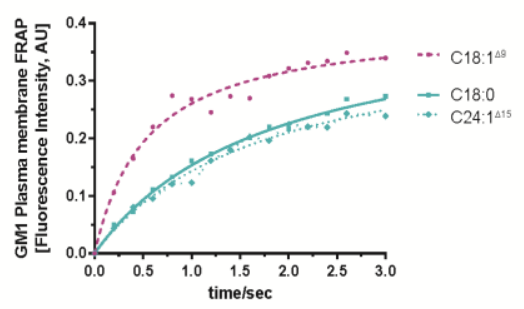

E.

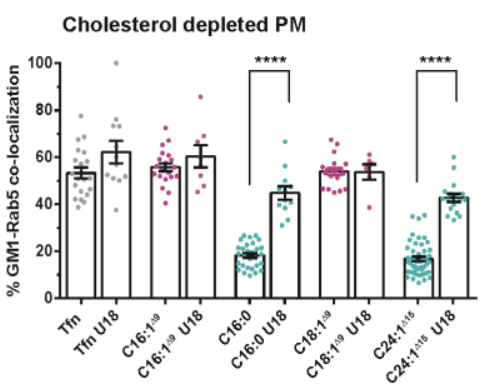

G.

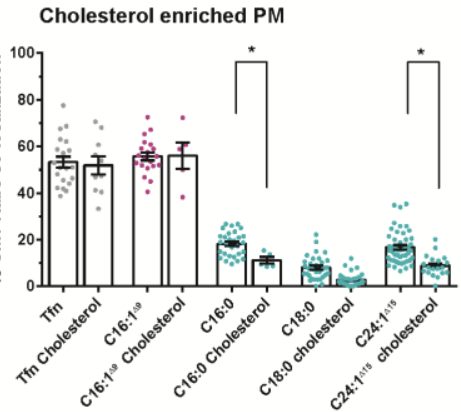

I.

973

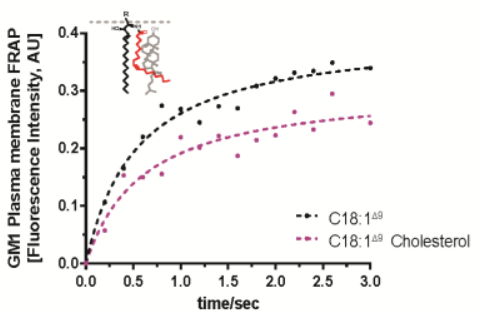

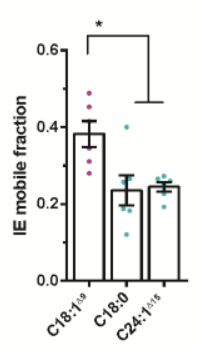

B.
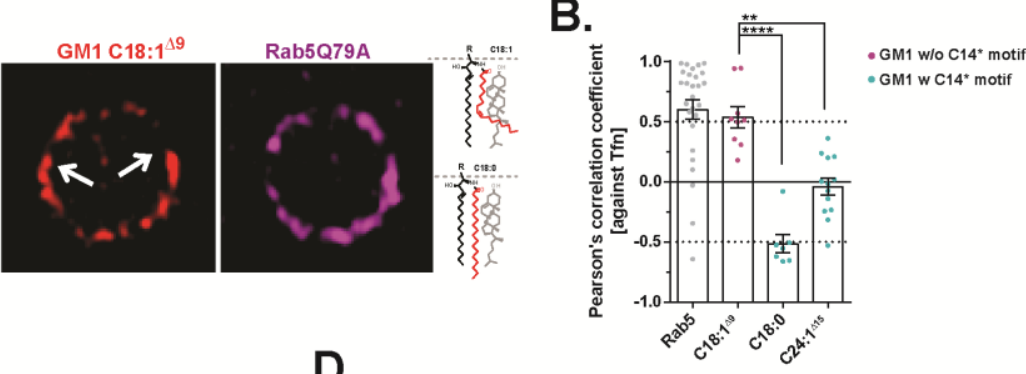

F.
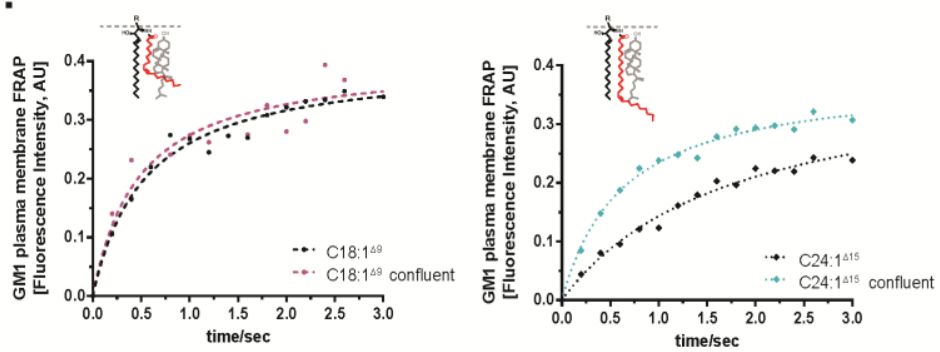

H.
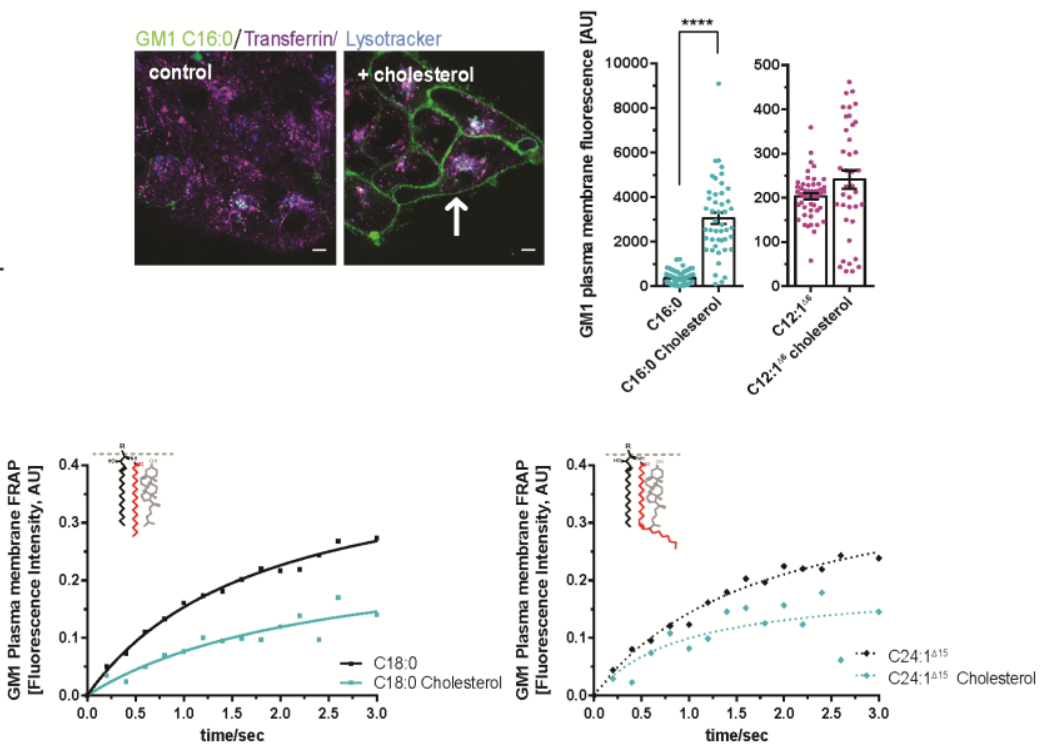
974 Fig. 4. C14* motif drives GM1 segregation in endosomes and at the plasma membrane. (A)

$975 \mathrm{GM} 1 \mathrm{C} 18: 0$ (green) and GM1 C18:1 $1^{\Delta 9}$ (red) were incorporated into A431 cells transfected with

976 dominant active RAB5Q79L-GFP plasmid (magenta). Scale bar = $1 \mu \mathrm{m}$. (B) Pearson's correlation

977 coefficient against Tfn. Dot represents Pearson's correlation coefficient for one enlarged RAB5

978 early endosome. GM1 species with C14* motif are displayed in turquoise and GM1 species

979 without $\mathrm{C} 14 *$ motif in magenta. ${ }^{* * * *} \mathrm{p}=<0.0001$ by One-way ANOVA and Tukey's multiple

980 comparison.. (C) A431 WT cells were incorporated with the respective GM1 species and a

981 plasma membrane ROI was photobleached immediately. Left panel: Fluorescence recovery

982 after bleaching (FRAP) was recorded and mean grey values of one representative experiment

983 are displayed. Right panel: Quantification of IE mobile fraction for FRAP experiments. Dot

984 represents mean grey fluorescence for one FRAP experiment. Mean \pm SEM. $* * * * p=<0.0001$

985 by One-way ANOVA and Tukey's multiple comparison. (D) Fluorescently labeled GM1 species

986 were incorporated into genome edited SVGA cells expressing RAB5-GFP. GM1 - RAB5-GFP

987 double positive vesicles were quantified. Mean \pm SEM. $* * * * p=<0.0001$ by One-way ANOVA

988 and Tukey's multiple comparison. (E) as in (D), but SVGA cells were mildly cholesterol depleted.

$989 * * * * \mathrm{p}=<0.0001$ by One-way ANOVA and Tukey's multiple comparison. (F) as in (C) but A431

990 cells were grown to over-confluency. (G) as in (D) but genome edited SVGA cells were

991 incorporated exogenous cholesterol. Mean \pm SEM. $* * * * p=<0.0001$ by One-way ANOVA and

992 Tukey's multiple comparison. (H) Left panel: GM1 C16:0 (green) was incorporated into HT29

993 cells. GM1 trafficking was observed $30 \mathrm{~min}$ post loading in control cells or cholesterol treated

994 cells. Scale bar $=1 \mu \mathrm{m}$. Right panel: Quantification of plasma membrane fluorescence of GM1

$995 \mathrm{C} 16: 0$ and $\mathrm{C} 12: 1^{\Delta 6}$ incorporated into HT29 WT cells untreated or treated with exogenous

996 cholesterol. Mean grey values of cell outlines for GM1 fluorescence were measured. Dot

997 represents mean grey fluorescence for one cell outline. Mean \pm SEM. ${ }^{* * * *} \mathrm{p}=<0.0001$ by One-

998 way ANOVA and Tukey's multiple comparison. (I) as in (C) but A431 cells were treated with

999 exogenous cholesterol. 


\section{Plasma and endocytic membrane at homeostasis}

a) GM1 fatty acid without $\mathrm{C} 14^{*}$ motif

- Cholesterol independent GM1 species - no evidence for nanodomain assembly

- high diffusive mobility in PM

- high levels of endocytosis

- enter recycling/retrograde tubules

- enable CTXB trafficking to ER

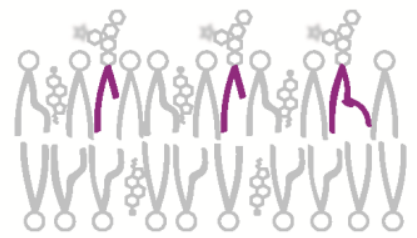

b) GM1 fatty acid with $\mathrm{C} 14^{*}$ motif

- Cholesterol dependent GM1 species

- evidence for nanodomain assembly

- low diffusive mobility in PM

- low levels of endocytosis

- sequestered within body of sorting endosome

- enable CTXB trafficking to lysosome

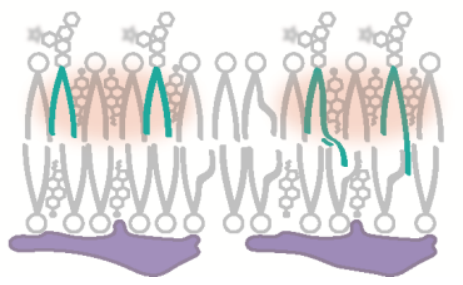

cholesterol

Phospholipid

GM1 without $\mathrm{C} 14^{*}$ motif

R

GM1 with $\mathrm{C} 14^{*}$ motif

nanodomain

cytoplasmic scaffolding protein

1001 Fig. 5. Model for cholesterol-ceramide structure based GM1 sorting. a) GM1 species with short or unsaturated acyl chains ( $\leq \mathrm{C} 14: 0$ or $\mathrm{C} 22: 1^{\Delta 13}$ ) lacking the $\mathrm{C} 14^{*}$ motif (magenta) are unable to

1003 assemble with cholesterol into membrane nanodomains. Their membrane and endocytic 1004 trafficking phenotypes are therefore independent of membrane cholesterol. b) GM1 species 1005 with long or very long unsaturated acyl chains ( $\geq \mathrm{C} 16: 0$ and $\left.\mathrm{C} 24: 1^{\Delta 15}\right)$ containing the $\mathrm{C} 14^{*}$ motif 1006 (turquoise) allow for close packing with cholesterol and therefore assembly into membrane 1007 nanodomains (or rafts). 
bioRxiv preprint doi: https://doi.org/10.1101/2021.04.26.440603; this version posted April 26, 2021. The copyright holder for this preprint (which was not certified by peer review) is the author/funder, who has granted bioRxiv a license to display the preprint in perpetuity. It is made available under ACC-BY-NC-ND 4.0 International license.

Supplementary Figures.

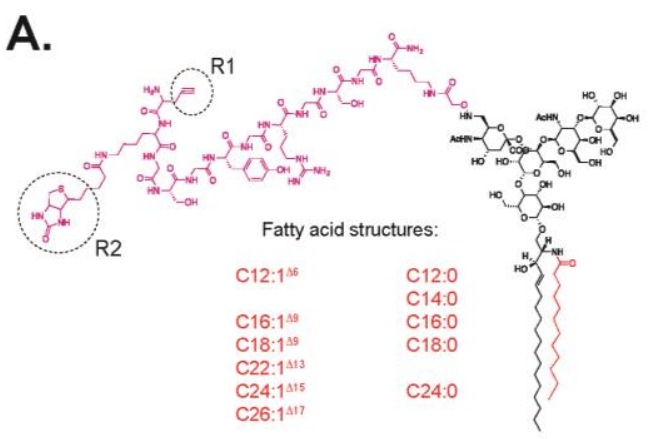

C.

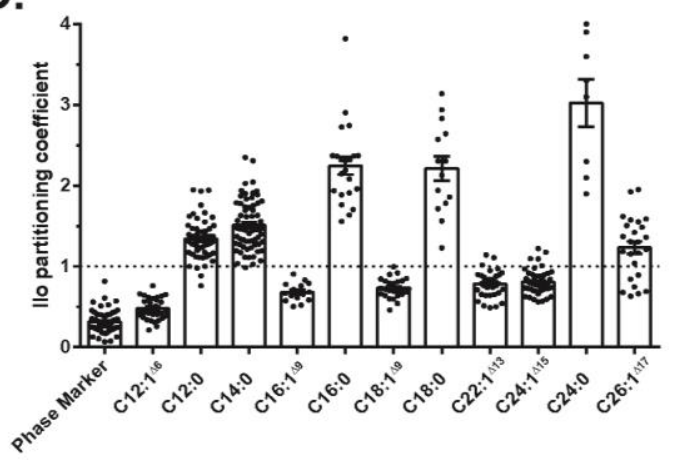

B.

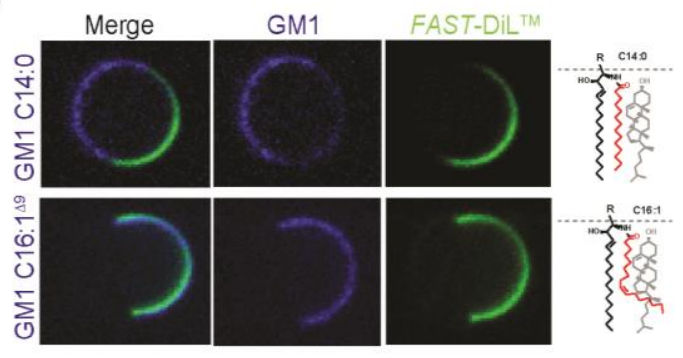

D.

\begin{tabular}{|c|c|c|c|}
\hline \multirow{2}{*}{ GM1 species } & \multicolumn{3}{|c|}{ Phase preference } \\
\hline & A431 & Hela & $\mathrm{CaCo}$ \\
\hline C12:1 & n.d. & $\mathrm{L}_{\mathrm{d}}$ & $\mathrm{L}_{d}$ \\
\hline C12:0 & NPP & NPP & $\mathrm{NPP}>\mathrm{L}$ \\
\hline C14:0 & NPP & $N P P>L$ 。 & $N P P>L$ \\
\hline $\mathrm{C} 16: 1^{10}$ & $\mathrm{~L}_{\sigma}$ & $L_{d}$ & $\mathrm{~L}_{d}$ \\
\hline C16:0 & $\mathrm{L}_{0}$ & n.d. & $L_{0}$ \\
\hline C18:1 $1^{18}$ & $\mathrm{~L}_{\mathrm{d}}$ & $\mathrm{L}_{\mathrm{c}}$ & $L_{d}$ \\
\hline C18:0 & n.d. & $L_{0}$ & $\mathrm{~L}_{0}$ \\
\hline $\mathrm{C} 22: 1^{1 / 3}$ & $L_{6}$ & $\mathrm{~L}_{d}$ & $\mathrm{~L}_{d}$ \\
\hline C24:1:15 & $L_{b}$ & $\mathrm{~L}_{\mathrm{d}}$ & $L_{d}$ \\
\hline C24:0 & n.d. & n.d. & $\mathrm{L}_{0}$ \\
\hline C26:1 & $\mathrm{L}_{\mathrm{d}}$ & $\mathrm{L}_{\mathrm{g}}$ & NPP \\
\hline
\end{tabular}

E.

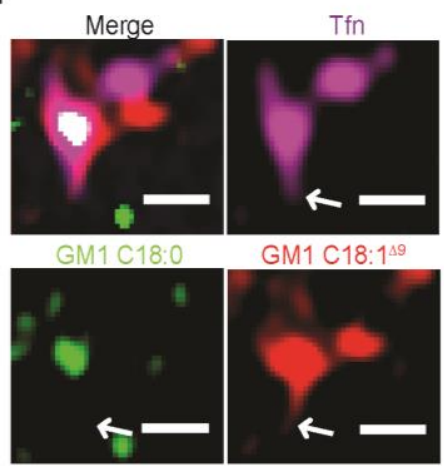

$\mathbf{F}$.

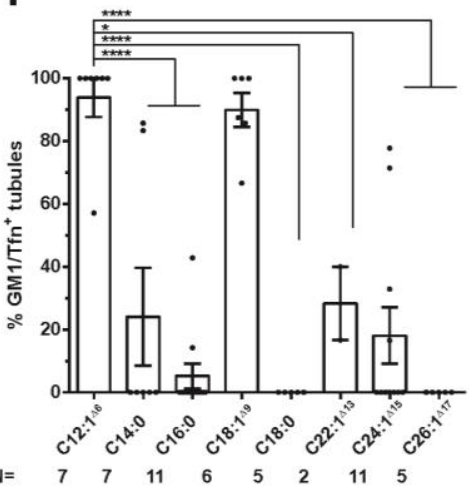

G.

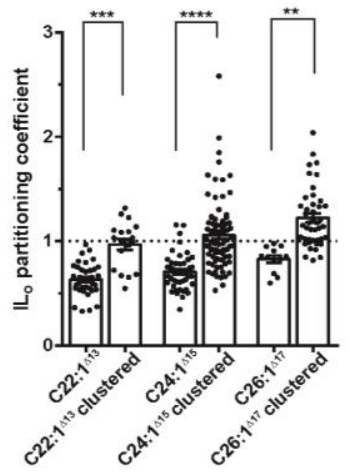

I.

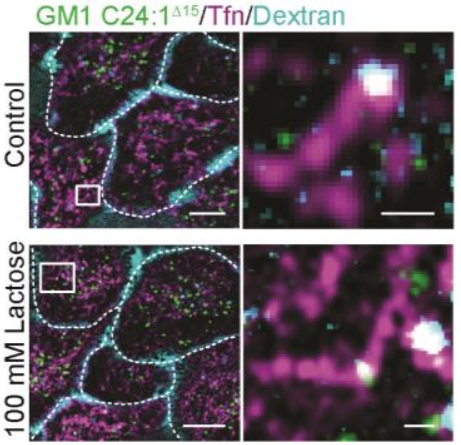

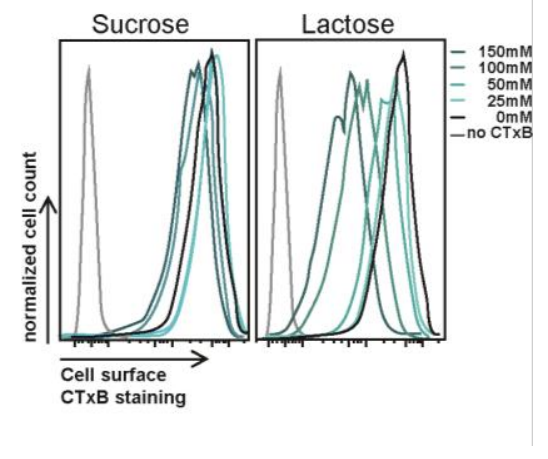




\section{Suppl. Fig. 1. Ceramide structure specific biophysical behavior and cellular sorting of GM1. (A)}

1013 Structure of the linker 'reporter' peptide (magenta) coupled to the sialic acid of GM1

1014 oligosaccharide (black) with functional biotin (R2) for streptavidin-AlexaFluor ${ }^{\mathrm{TM}}$ detection and 1015 alkyne group (R1) for direct AlexaFluor ${ }^{\mathrm{TM}}$ azide conjugation. Sialic acid is also used for direct 1016 AlexaFluor ${ }^{\mathrm{TM}}$ - GM1 conjugates. Different fatty acyl chain structures used in the study are 1017 displayed in red. (B) GPMV from CaCo BBE WT cells loaded with fluorescent GM1 C14:0 and $1018 \mathrm{C} 16: 1^{\Delta 9}$ and FAST-DiL ${ }^{\mathrm{TM}}$ phase marker. Exemplary images shown. (C) Phase preference (IL。 1019 partitioning coefficient) for the library of GM1 species. Dot represents $\mathrm{IL}_{\mathrm{o}}$ partitioning 1020 coefficient for one GPMV. Dotted line represents switch between $I L_{D}$ and $I L_{0}$ phase preference. $1021 \mathrm{~N}=\geq 8$ experiments. (D) Summary table for phase preference in the different cell lines. (E) GM1 $1022 \mathrm{C} 18: 0$ (green) and GM1 C18:1 ${ }^{\Delta 9}$ (red) were concomitantly incorporated into the plasma 1023 membrane of Hela cells. Scale bar $=1 \mu \mathrm{m}$. (F) Quantification of GM1 presence in Tfn positive 1024 recycling endosomal tubules of Hela cells as for Fig. 1E. Mean \pm SEM. ${ }^{* * * *} p=<0.0001$ by One1025 way ANOVA and Tukey's multiple comparison. (G) Phase preference of different GM1 species, 1026 directly fluorescently labeled or clustered by fluorescently labeled CTxB. Dot represents IL。 1027 partitioning coefficient of 1 GPMV, $\mathrm{n}=\geq 4$ experiments. $* * * * \mathrm{p}=<0.0001$ by One-way ANOVA 1028 and Tukey's multiple comparison. (H) Endosomal sorting of GM1 C16:1 ${ }^{\Delta 9}$ or GM1 C24:1 $1^{\Delta 15}$ 1029 (green) was monitored in A431 cells in the presence or absence of $100 \mathrm{mM}$ lactose, and 1030 transferrin (magenta) and dextran (blue) as in Fig.1C. Scale bars $=5 \mu \mathrm{m}$ and $0.5 \mu \mathrm{m}$ respectively. 1031 (I) FACS sorting of A431 cells incubated with $5 \mathrm{nM}$ fluorescently labeled CTxB in the presence of 1032 indicated amount of sucrose or lactose. Cells were then FACS sorted to measure CTxB binding 1033 to the cell surface. Lines represent fluorescence of 20,000 cells sorted. 
A.
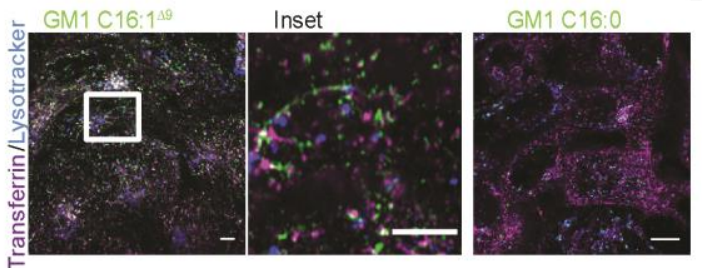

B.

C.

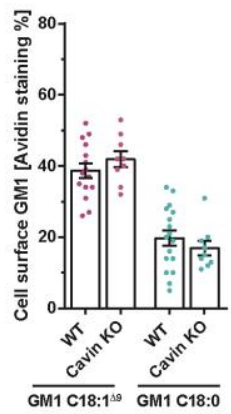

D.
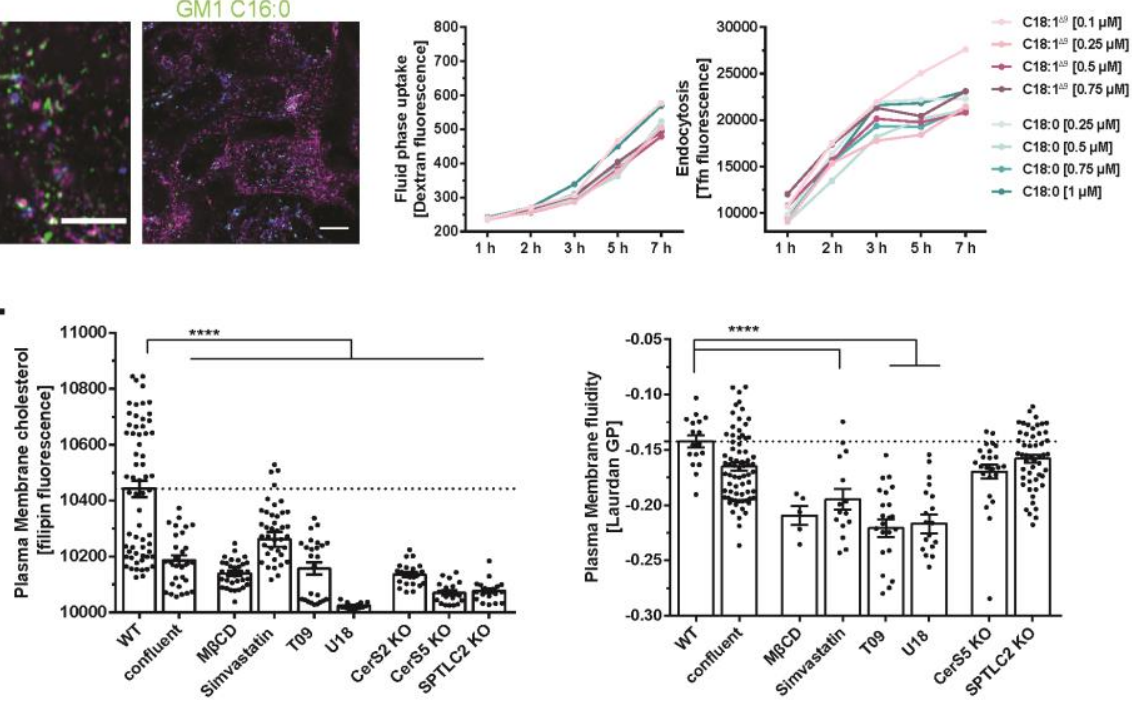

E.
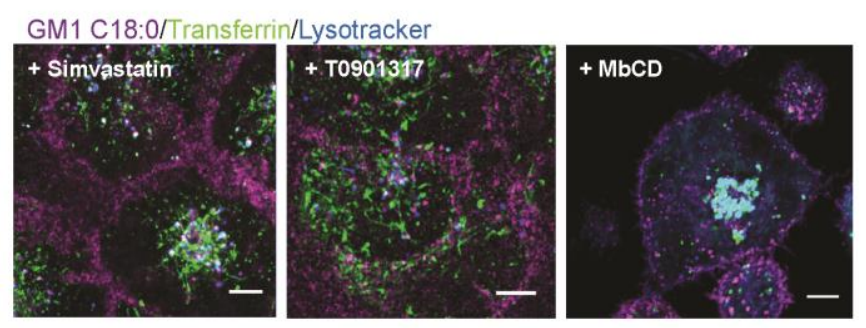

F.

Recycling GM1 species

\begin{tabular}{|c|c|c|c|c|c|c|}
\hline \multirow{2}{*}{$\begin{array}{l}\text { GM1 } \\
\text { species }\end{array}$} & \multicolumn{6}{|c|}{$\begin{array}{c}\text { Fold change cell surface } \\
\text { chase cholesterol depletion/control] }\end{array}$} \\
\hline & T09 & U18 & $\begin{array}{l}\text { over - } \\
\text { confluent }\end{array}$ & $\underset{\text { KO }}{\text { SPTLC2 }}$ & CerS2 Ko & CerS5 KO \\
\hline C12:0 & $1.01+0.1$ & $1.14+0.1$ & $0.88+0.1$ & $0.80+0.1$ & $0.83+0.2$ & $0.86+0.1$ \\
\hline C14:0 & $0.93+0.1$ & $1.15+0.1$ & $1.03+0.2$ & $0.87+0.4$ & $0.96+0.2$ & $0.91+0.5$ \\
\hline$C 16: 1^{19}$ & $0.94+0.1$ & $0.98+0.2$ & $0.87+0.1$ & $0.80+0.1$ & $1.00+0.2$ & $0.85+0.1$ \\
\hline C18:199 & $0.92+0.2$ & $1.07+0.1$ & $0.91+0.2$ & $0.89+0.1$ & $1.02+0.1$ & $1.08+0.1$ \\
\hline C22:1413 & $1.19+0.6$ & $1.28+0.3$ & $1.32+0.3$ & $1.24+0.2$ & $1.33+0.2$ & $1.41+0.2$ \\
\hline
\end{tabular}

Lysosomal GM1 species $\uparrow$

\begin{tabular}{|c|c|c|c|c|c|c|}
\hline \multirow{2}{*}{$\begin{array}{l}\text { GM1 } \\
\text { species }\end{array}$} & \multicolumn{6}{|c|}{$\begin{array}{c}\text { Fold change cell surface GM1 } \\
\text { [chase cholesterol depletion/control] }\end{array}$} \\
\hline & T09 & U18 & $\begin{array}{l}\text { over - } \\
\text { confluent }\end{array}$ & $\underset{\text { KO }}{\text { SPTLC } 2}$ & CerS2 KO & CerS5 Ko \\
\hline C16:0 & $1.56+0.2$ & $1.84+0.3$ & $1.59+0.1$ & $1.52+0.1$ & $1.33+0.2$ & $1.40+0.1$ \\
\hline C18:0 & $1.45+0.2$ & $1.82+0.2$ & $1.56+0.2$ & $1.64+0.3$ & $1.41+0.1$ & $1.57+0.2$ \\
\hline $\mathrm{C} 24: 1^{1 / 15}$ & $1.47+0.2$ & $2.19+0.3$ & $1.54+0.3$ & $1.32+0.2$ & $1.49+0.2$ & $1.46+0.1$ \\
\hline C26:1 $1^{117}$ & $1.55+0.4$ & $2.08+0.3$ & $1.35+0.3$ & $1.36+0.2$ & $1.50+0.2$ & $1.51+0.2$ \\
\hline
\end{tabular}

G.

H.

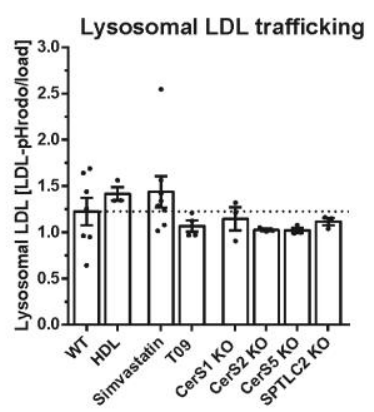

\begin{tabular}{|c|c|c|}
\hline \multirow[t]{2}{*}{ GM1 species } & \multicolumn{2}{|c|}{ Phase preference } \\
\hline & $50 \%$ confluent & over-confluent \\
\hline C12:1 & $L_{d}$ & $L_{d}$ \\
\hline C12:0 & $N P P>L_{0}$ & $N P P>L_{0}$ \\
\hline C14:0 & $N P P>L_{0}$ & $N P P>L_{0}$ \\
\hline $\mathrm{C} 16: 1^{19}$ & $L_{d}$ & $L_{d}$ \\
\hline C16:0 & Lo & $L_{0}$ \\
\hline C18:1 & $L_{d}$ & $L_{d}$ \\
\hline C18:0 & Lo & $L_{0}$ \\
\hline $\mathrm{C} 22: 1^{\wedge 13}$ & $L_{d}$ & $L_{d}$ \\
\hline $\mathrm{C} 24: 1^{\$ 15}$ & Ld & $L_{d}$ \\
\hline C24:0 & $L_{0}$ & $L_{0}$ \\
\hline C26:1 & NPP & NPP \\
\hline
\end{tabular}

1036 Suppl. Fig. 2. Cholesterol dependent sorting into lysosomal pathway. (A) Flu GM1 C16:1 ${ }^{19}$

1037 (green) and C16:0 (green) were loaded into A431 WT cells and trafficked with transferrin 
1038 (magenta) and lysotracker (blue) for $6 \mathrm{~h}$. Recycling endosomal tubules were visualized (Inset).

1039 Scale bar $=5$ and $1 \mu \mathrm{m}$ respectively. (B) Different concentrations of GM1 C18:1 $1^{\Delta 9}$ and GM1

1040 C18:0 were incorporated into A431 cells. GM1 species with C14* motif are displayed in

1041 turquoise and GM1 species without C14* motif in magenta. Transferrin and dextran were

1042 added during the 'chase' period to monitor fluid phase uptake by FACS. Dot represents mean of

10432 biological experiments measuring 20,000 cells each. (C) GM1 C18:1 ${ }^{\Delta 9}$ and GM1 C18:0

1044 recycling was measured in A431 WT cells or CRISPR-Cas9 KO cells for CAVIN-1 as in (Fig. 1A).

1045 Dot represents mean of 2 biological experiments measuring 20,000 cells each. Unpaired t-test

1046 was performed. (D) A431 WT control cells and cells treated to mildy lower cholesterol were

1047 fixed and stained for plasma membrane cholesterol quantification using filipin III (left panel).

1048 Cell outlines were detected and mean grey values plotted. Right panel: Membrane fluidity was

1049 measured in GPMVs obtained from control and treated cells using laurdan. Each dot represents

1050 the general polarization (GP) value for one GPMV. Mean and SEM. ${ }^{* * * *} \mathrm{p}=<0.0001$ by One-

1051 way ANOVA and Tukey's multiple comparison. (E) GM1 C18:0 (magenta) was incorporated into

1052 A431 cells treated as indicated. Tfn (green) and lysotracker ${ }^{\mathrm{TM}}$ (blue) were added to demark

1053 integrity of intracellular organelles. (F) Tabularized summary of plasma membrane recycling for

1054 different GM1 species under respective cholesterol lowering conditions as in Fig. 2D. Fold

1055 change of GM1 amount at the plasma membrane in treated over control conditions are

1056 displayed. ${ }^{* *} \mathrm{p}=<0.01$ or n.s. by One-way ANOVA and Tukey's multiple comparison. (G)

1057 Lysosomal trafficking of pHrodo $^{\mathrm{TM}}$-labeled LDL by FACS for A431 cells untreated or treated as

1058 indicated (as in D and F). Dot represents mean of 2 biological experiments with 20,000 cells

1059 each. Mean \pm SEM. One-way ANOVA and Tukey's multiple comparison. $(\mathbf{H})$ Phase preference

1060 was quantified different GM1 species in A431 cell GPMVs at 50\% confluency or grown to over-

1061 confluency for $48 \mathrm{~h}$. 

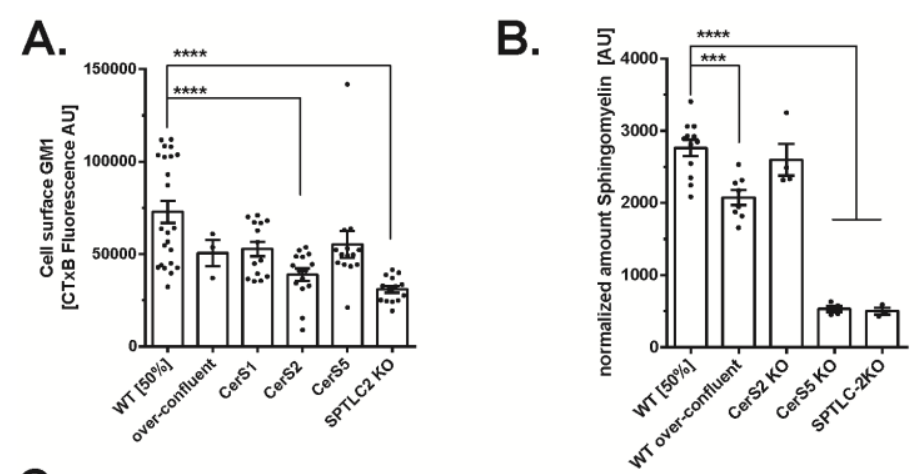

C.

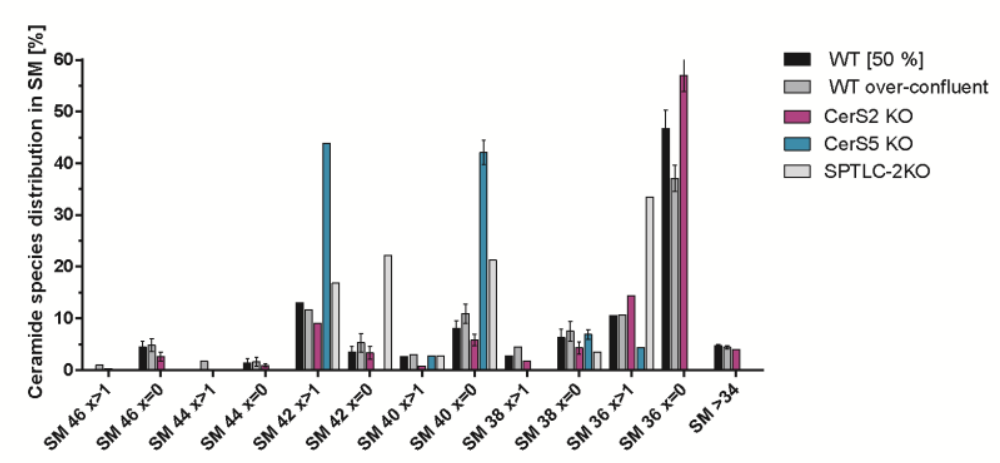

D.
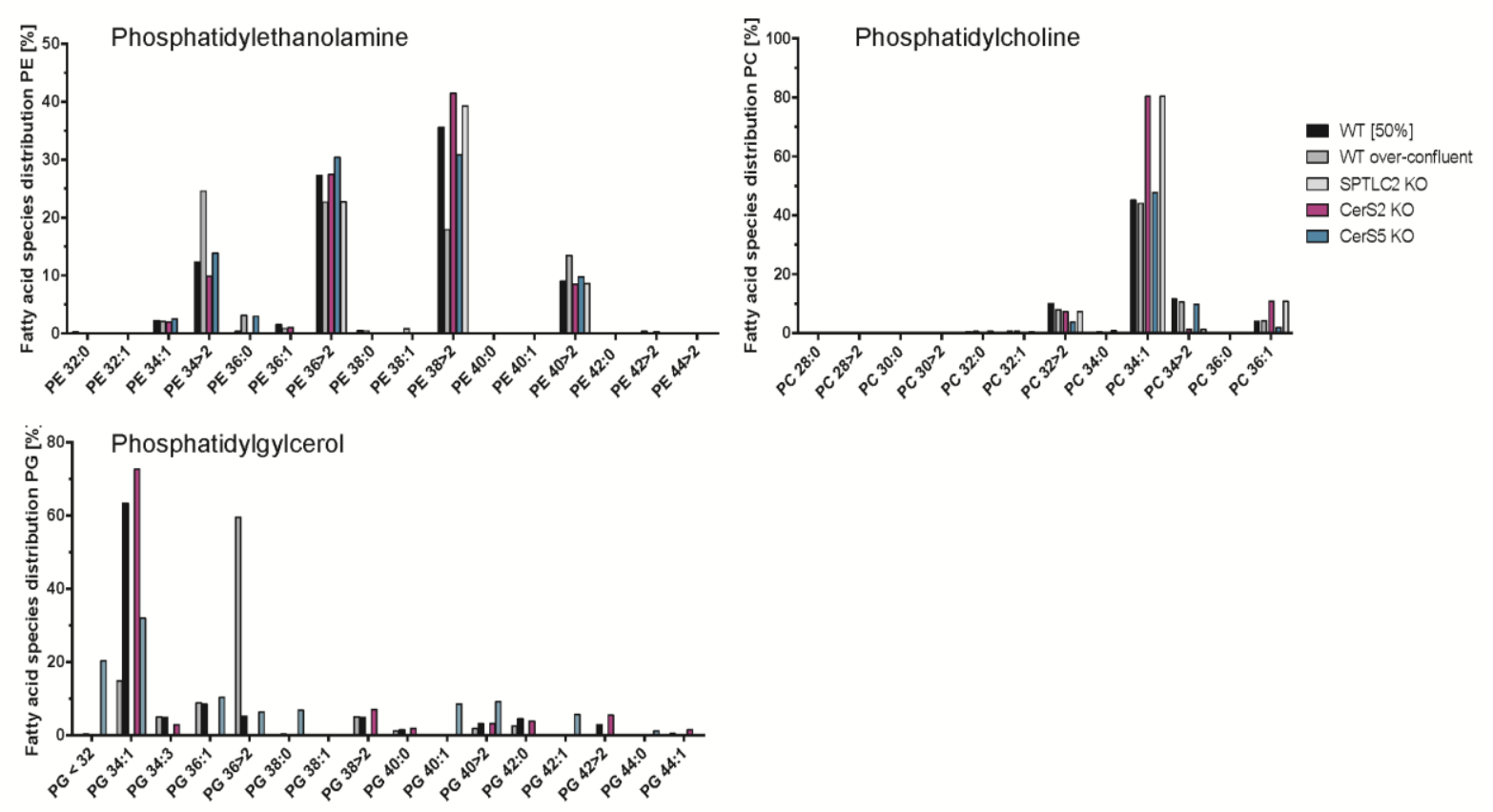

1063 Suppl. Fig. 3. Lipidomic profiling of A431 WT and ceramide synthase KO cell lines. (A) CTxB surface staining for GM1 amount in A431 WT or CRISPR-Cas9 KO as indicated. Mean \pm SEM. Dot represents mean of 2 biological experiments measuring 20,000 cells each. $* * * * p=<0.0001$ by 
1067 profiling for A431 WT, over-confluent and CRISPR-Cas9 KO as indicated. Mean \pm SEM. $* * * * p=$

$1068<0.0001$ or $* * * \mathrm{p}=<0.001$ by One-way ANOVA and Tukey's multiple comparison. (C) Ceramide

1069 profile of sphingomyelin species in \% for A431 WT, over-confluent and CRISPR-Cas9 KO as

1070 indicated. $\mathrm{N}=>4$ biological replicates. (D) Phosphatidylcholine (PC), - glycerol (PG) and -

1071 ethanolamine (PE) fatty acid profiles in \% for A431 WT, over-confluent and CRISPR-Cas9 KO as

1072 indicated.

A.

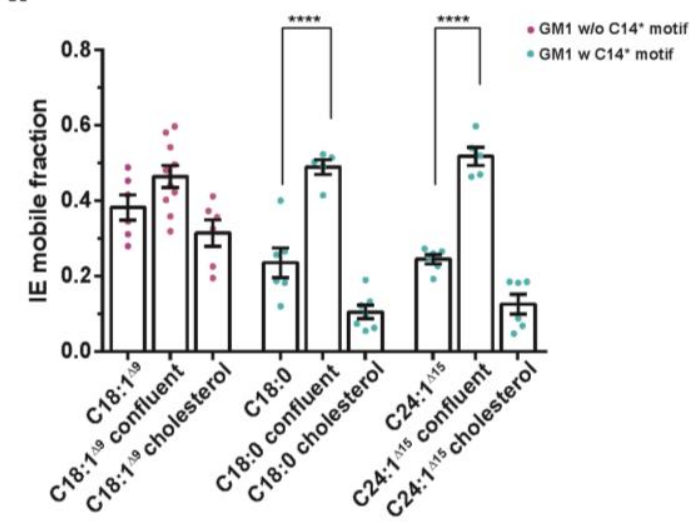

D.
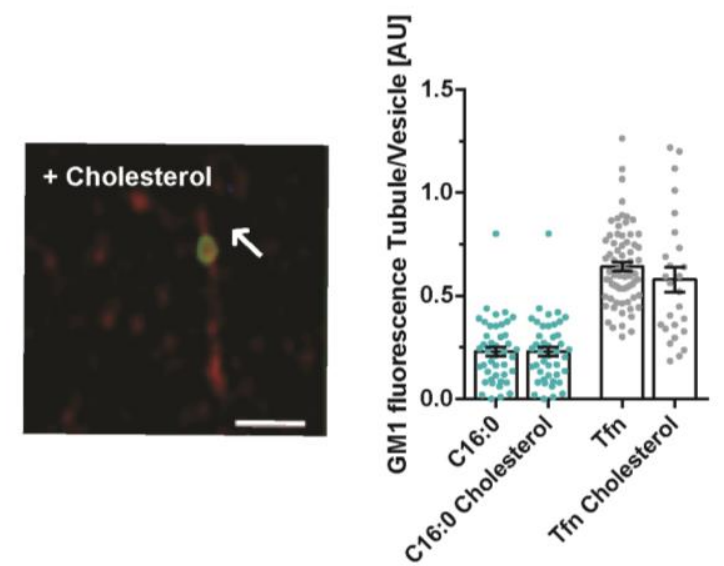

F.
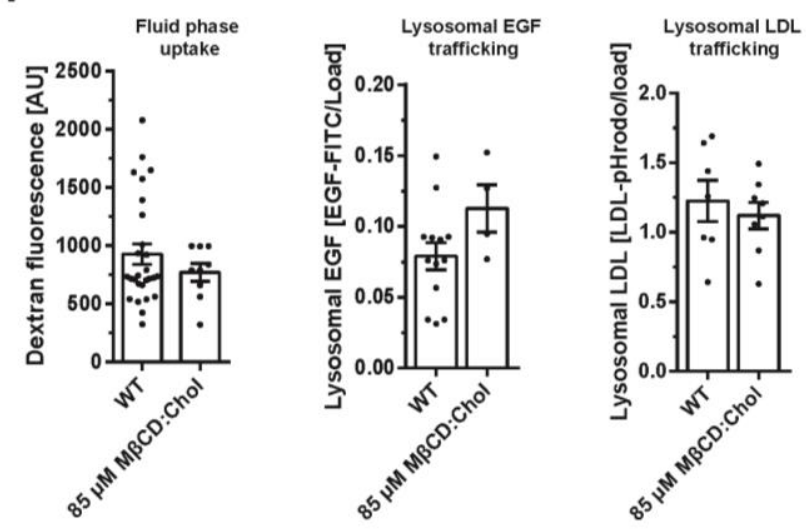

B.
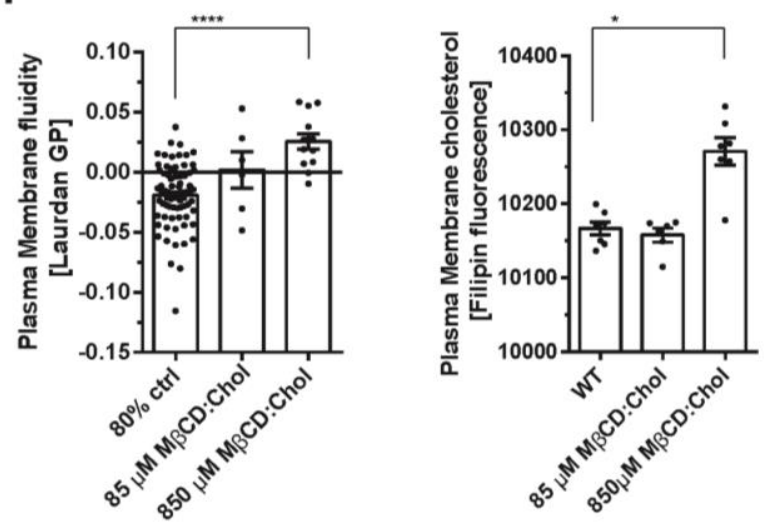

E.

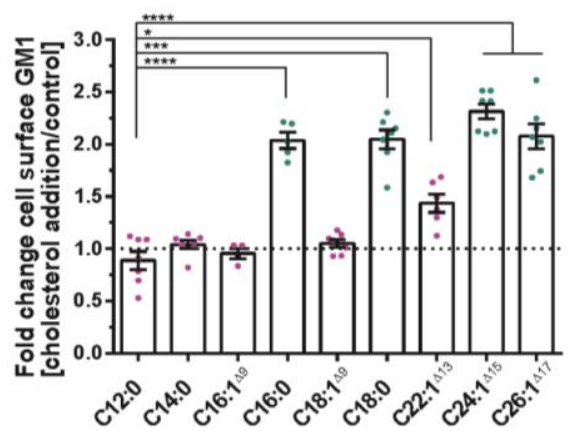


1074 Suppl. Fig. 4. Plasma membrane nanodomain behavior of different GM1 species. (A) 1075 Quantification of IE mobile fraction for FRAP experiments in Fig. 4C, F and I. Dot represents 1076 mean grey fluorescence for one FRAP experiment. Mean \pm SEM. $* * * * p=<0.0001$ by One-way 1077 ANOVA and Tukey's multiple comparison. (B) Left panel: Filipin staining of plasma membranes 1078 of A431 WT cell treated with exogenous cholesterol or untreated. Right panel: Laurdan staining 1079 of GPMVs produced from A431 control or cholesterol treated A431 cells Dot represents mean 1080 of 2 biological replicates or one GPMV respectively. Mean \pm SEM. $* * * * p=<0.0001$ or $* p=<$ 10810.05 by One-way ANOVA and Tukey's multiple comparison. (D) Left panel: GM1 C16:0 (green) 1082 and transferrin (red) were added to A431 cells treated with exogenous cholesterol. Right panel: 1083 Ratio of GM1 C16:0 and Tfn in recycling endosomal tubules over its respective vesicle. Dots 1084 represent 1 tubule to vesicle ratio. Mean \pm SEM. One-way ANOVA and Tukey's multiple 1085 comparisons were performed. (E) FACS based recycling assay for linker-peptide labeled GM1 1086 species in cholesterol treated A431 cells. Fold change between treatment and control is 1087 plotted. Dot represents mean of two biological replicates measuring 20,000 cells. Mean \pm SEM. $1088 * * * * \mathrm{p}=<0.0001, * * * \mathrm{p}=<0.001, * \mathrm{p}=<0.05$ by One-way ANOVA and Tukey's multiple 1089 comparison. (F) Left panel: dextran fluid phase uptake by FACS was measured in A431 WT cells 1090 treated with exogenous cholesterol or untreated. Center and right panel: Lysosomal EGF and 1091 LDL trafficking in A431 WT cells treated with exogenous cholesterol or untreated using FITC-EGF 1092 and $\mathrm{pHrodo}^{\mathrm{TM}}$-LDL. Dot represents mean of two biological replicates measuring 20,000 cells. 1093 Mean \pm SEM. One-way ANOVA and Tukey's multiple comparisons were performed to test 1094 statistical difference. ${ }^{* * * *} \mathrm{p}=<0.0001, * * * \mathrm{p}=<0.001, * \mathrm{p}=<0.05$ by One-way ANOVA and 1095 Tukey's multiple comparison.

\section{Supplementary Video Files}

1098 Suppl. Video1. Lattice lightsheet microscopy of sorting endosome containing GM1 C16:1 ${ }^{\Delta 9}$ 1099 (green), transferrin (magenta) and dextran (turquoise) with sorting tubules emerging. 
bioRxiv preprint doi: https://doi.org/10.1101/2021.04.26.440603; this version posted April 26, 2021. The copyright holder for this preprint (which

was not certified by peer review) is the author/funder, who has granted bioRxiv a license to display the preprint in perpetuity. It is made available under aCC-BY-NC-ND 4.0 International license.

1100 Suppl. Video1. Lattice lightsheet microscopy of sorting endosome containing GM1 C18:1 ${ }^{\Delta 9}$

1101 (green), and GM1 C18:0 (magenta) with sorting tubules emerging.

1102

1103

1104 